NBER WORKING PAPER SERIES

\title{
A GENERALIZED APPROACH TO INDETERMINACY IN LINEAR RATIONAL EXPECTATIONS MODELS
}

Francesco Bianchi

Giovanni Nicolò

Working Paper 23521

http://www.nber.org/papers/w23521

\author{
NATIONAL BUREAU OF ECONOMIC RESEARCH \\ 1050 Massachusetts Avenue \\ Cambridge, MA 02138 \\ June 2017
}

We thank Jonas Arias, Jess Benhabib, Roger Farmer, François Geerolf, Frank Schorfheide and all participants at UCLA seminars, NBER Multiple Equilibria and Financial Crises Conference, CEPR-IMFS New Methods for Macroeconomic Modeling, Model Comparison and Policy Analysis Conference, Federal Reserve Bank of St. Louis, Society of Economic Dynamics, 12th Dynare Conference, 2017 NBER-NSF conference on Bayesian Inference in Econometrics and Statistics. The views expressed herein are those of the authors and do not necessarily reflect the views of the National Bureau of Economic Research.

NBER working papers are circulated for discussion and comment purposes. They have not been peer-reviewed or been subject to the review by the NBER Board of Directors that accompanies official NBER publications.

(C) 2017 by Francesco Bianchi and Giovanni Nicolò. All rights reserved. Short sections of text, not to exceed two paragraphs, may be quoted without explicit permission provided that full credit, including $\odot$ notice, is given to the source. 
A Generalized Approach to Indeterminacy in Linear Rational Expectations Models

Francesco Bianchi and Giovanni Nicolò

NBER Working Paper No. 23521

June 2017

JEL No. C19,C51,C62,C63

\begin{abstract}
We propose a novel approach to deal with the problem of indeterminacy in Linear Rational Expectations models. The method consists of augmenting the original model with a set of auxiliary exogenous equations that are used to provide the adequate number of explosive roots in presence of indeterminacy. The solution in this expanded state space, if it exists, is always determinate, and is identical to the indeterminate solution of the original model. The proposed approach accommodates determinacy and any degree of indeterminacy, and it can be implemented even when the boundaries of the determinacy region are unknown. As a result, the researcher can estimate the model by using standard packages without restricting the estimates to a certain area of the parameter space. We apply our method to simulated and actual data from a prototypical New-Keynesian model for both regions of the parameter space. We show that our method successfully recovers the true parameter values independent of the initial values.
\end{abstract}

Francesco Bianchi

Social Sciences Building, 201B

Department of Economics

Duke University

Box 90097

Durham, NC 27708-0097

and CEPR

and also NBER

francesco.bianchi@duke.edu

Giovanni Nicolò

UCLA

Department of Economics

Los Angeles, CA 90095-1477

gnicolo@ucla.edu 


\section{Introduction}

Sunspot shocks and multiple equilibria have been at the center of economic thinking at least since the seminal work of Cass and Shell (1983), Farmer and Guo (1994) and Farmer and Guo (1995). Furthermore, in many of the Linear Rational Expectation (LRE) models used to study the properties of the macroeconomy the possibility of multiple equilibria arises for some parameter values, but not for others. This paper proposes a novel approach to solve LRE models that easily accommodates both the case of determinacy and indeterminacy. As a result, the proposed methodology can be used to easily estimate a LRE model that could potentially be characterized by multiplicity of equilibria. Our approach is implementable even when the analytic conditions for determinacy or the degrees of indeterminacy are unknown and can be implemented to study indeterminacy in standard software packages, such as Dynare and Sims' (2001) code Gensys.

To understand how our approach works, it is useful to recall the conditions for determinacy as stated by Blanchard and Kahn (1980). Indeterminacy arises when the parameter values are such that the number of explosive roots is smaller than the number of non-predetermined variables. The key idea behind our methodology consists of augmenting the original model by appending additional autoregressive processes that can be used to provide the missing explosive roots. The innovations of these exogenous processes are assumed to be linear combinations of a subset of the forecast errors associated with the expectational variables of the model, and a newly defined vector of sunspot shocks. Whether the autoregressive processes are mean-reverting or explosive is central, and the intuition follows. When a model is determinate, the roots of the additional autoregressive process are within the unit circle (i.e., the Blanchard-Kahn condition is satisfied) and the auxiliary process is irrelevant for the dynamics of the model. The law of motion for the endogenous variables is in this case equivalent to the solution obtained using standard solution algorithms (King and Watson (1998), Klein (2000), Sims (2001)). When the model is indeterminate, the appended autoregressive processes are explosive, and the solution we obtain for the endogenous variables is equivalent to the one obtained with the methodology of Lubik and Schorfheide (2003) or, equivalently, Farmer et al. (2015).

Our methodology simplifies the common approach used to deal with indeterminacy. First, the common procedure requires the researcher to solve the model differently depending on the area of the parameter space that is being studied. Second, the procedure requires to estimate the same model twice, first under determinacy, then under indeterminacy. This is the same procedure that would be followed if the researcher were comparing two structurally different models, while she is in fact estimating the same structural model in alternative regions of the parameter space. Finally, the estimation under indeterminacy is not generally implementable in standard estimation packages and requires a significant amount of coding work on the side of the researcher. 
In this respect, our methodology provides three main advantages. First, it accommodates both the case of determinacy and indeterminacy while considering the same augmented system of equations. The model can therefore be solved by using standard solution algorithms. Instead, existing methods require to rewrite the model based on the existing degree of indeterminacy (Farmer et al. (2015)) or to construct the solution under indeterminacy ex-post following the seminal contribution of Lubik and Schorfheide (2003). Second, given that the method accommodates both the case of determinacy and indeterminacy, the researcher does not need to take a stance on which area of the parameter space she is interested in exploring. We show that our methodology ensures that standard estimation algorithms explore the entire parameter space, increasing the probability of finding a global maximum over the parameter space. This is particularly relevant when considering that the posterior mode is a crucial object used for Bayesian inference. ${ }^{1}$ Finally, even when the region of determinacy is unknown, the methodology allows the researcher to estimate the model without imposing a priori assumptions about the uniqueness of the equilibrium, which can be equivalently thought of as restrictions on the parameter space over which inference is conducted. Hence, information contained in the data indicates whether an estimated model is characterized by a unique solution or by multiplicity of equilibria.

Our work is related to the vast literature that studies the role of indeterminacy in explaining the evolution of the macroeconomy. Prominent examples in the monetary policy literature include the work of Clarida et al. (2000) and Kerr and King (1996), that study the possibility of multiple equilibria as a result of violations of the Taylor principle in New-Keynesian (NK) models. Applying the methods developed in Lubik and Schorfheide (2003) to the canonical NK model, Lubik and Schorfheide (2004) test for indeterminacy in U.S. monetary policy. Using a calibrated small-scale model, Coibon and Gorodnichenko (2011) find that the reduction of the target inflation rate in the U.S. also played a key role in explaining the Great Moderation, and Arias et al. (2015) support this finding in the context of a medium-scale model $\grave{a}$ la Christiano et al. (2005). In a similar spirit, Arias (2013) studies the dynamic properties of medium-sized NK models with trend inflation. More recently, Aruoba and Schorfheide (2015) study inflation dynamics at the Zero Lower Bound (ZLB) and during an exit from the ZLB.

The paper closest to our is Farmer et al. (2015). As explained above, the main difference between the two approaches is that our method accommodates both the case of determinacy and indeterminacy while considering the same augmented system of equations. Instead, the method proposed by Farmer et al. (2015) require to rewrite the model based on the existing degree of indeterminacy. With respect to Lubik and Schorfheide (2003), the main novelty of our approach

\footnotetext{
${ }^{1}$ Specifically, using simulated data we show that our methodology leads the estimation algorithm to converge to the "right" area of the parameter space. Once the algorithm converges to such area, the probability of leaving it is very low, in line with the results of Lubik and Schorfheide (2004) that show that the likelihood presents potentially very large jumps/drops between the determinacy/indeterminacy regions.
} 
is to provide a unified approach to study determinacy and indeterminacy of different degrees. Ascari et al. (2016) allow for temporarily unstable paths, while we require all solutions to be stationary, in line with previous contributions in the literature. Finally, we deliberately use Dynare in all the examples presented in this paper to show that our method can be combined with standard packages. However, our solution method can be combined with more sophisticated estimation techniques such as the ones developed in Herbst and Schorfheide (2015).

The remainder of the paper is organized as follows. Section 2 builds the intuition by using a univariate example in the spirit of Lubik and Schorfheide (2004). Section 3 describes the methodology and shows that the augmented representation of the LRE model delivers solutions which under determinacy are equivalent to those obtained using standard solution algorithms, and under indeterminacy to those obtained using the methodology provided by Lubik and Schorfheide (2003, 2004) and Farmer et al. (2015). Section 4 provides an analytic example of the theoretical result and in Section 5, we apply our theoretical results to the NK model of Lubik and Schorfheide (2004). In Section 5.1, we first generate series of simulated data for parameter values which satisfy the condition for determinacy and indeterminacy, respectively. We then estimate the model by using the proposed augmented representation for both cases. The model is estimated over the

entire parameter space and the true parameter values are recovered, providing evidence in favor of determinacy or indeterminacy. Section 5.2 shows that this is true even when we assume that the researcher does not know the boundaries of the determinacy region. Hence, our methodology can be used to test for indeterminacy in a wide class of models, including medium- and largescale models for which the region of determinacy cannot be derived analytically. We also repeat the exercise on actual data using the dataset from Lubik and Schorfheide (2004) in Section 5.3. Finally, we provide guidance on how to properly implement our methodology in Section 6 and our conclusions are presented in Section 7.

\section{Building the intuition}

Before presenting the theoretical results of the paper, this section builds the intuition behind our approach by considering a univariate example similar to the one proposed in Lubik and Schorfheide (2004). While Section 2.1 explains our approach from an analytical perspective, Section 2.2 addresses questions which could arise at the time of its practical implementation. 


\subsection{A useful example}

Consider a classical monetary model characterized by the Fisher equation

$$
i_{t}=E_{t}\left(\pi_{t+1}\right)+r_{t}
$$

and the simple Taylor rule

$$
i_{t}=\phi_{\pi} \pi_{t}
$$

where $i_{t}$ denotes the nominal interest rate. We assume that the real interest rate $r_{t}$ is given and described by a mean-zero Gaussian i.i.d. shock. ${ }^{2}$ To properly specify the model, we also define the one-step ahead forecast error associated with the expectational variable, $\pi_{t}$, as

$$
\eta_{t} \equiv \pi_{t}-E_{t-1}\left(\pi_{t}\right)
$$

Combining (1) and (2), we obtain the univariate model

$$
E_{t}\left(\pi_{t+1}\right)=\phi_{\pi} \pi_{t}-r_{t}
$$

First, we consider the case $\phi_{\pi}>1$. Rewriting equation (4), it is clear that this case is associated with the determinate solution,

$$
\begin{aligned}
\pi_{t} & =\frac{1}{\phi_{\pi}} E_{t}\left(\pi_{t+1}\right)+\frac{1}{\phi_{\pi}} r_{t} \\
& =\frac{1}{\phi_{\pi}} r_{t} .
\end{aligned}
$$

where the last equality is obtained by solving equation (5) forward and recalling the assumptions on $r_{t}$. The strong response of the monetary authority to changes in inflation $\left(\phi_{\pi}>1\right)$ guarantees that inflation is pinned down as a function of the exogenous real interest $r_{t}$. From a technical perspective, the condition $\phi_{\pi}>1$ is such that the Blanchard-Kahn condition is satisfied: the number of explosive roots matches the number of expectational variables, that in this univariate case is $\pi_{t}$.

The second case corresponds to $\phi_{\pi} \leq 1$. The solution to (4) is obtained by combining (4) with

\footnotetext{
${ }^{2}$ In the classical monetary model, the real interest rate results from the equilibrium in labor and goods market and it depends on the technology shocks. We are considering an exogenous process for the technology shocks and therefore we take the process for the real interest rate as given.
} 
(3), and it corresponds to any process that takes the following form

$$
\pi_{t}=\phi_{\pi} \pi_{t-1}-r_{t-1}+\eta_{t}
$$

When the monetary authority does not respond aggressively enough to changes in inflation $\left(\phi_{\pi} \leq 1\right)$, there are multiple solutions for the inflation rate, $\pi_{t}$, each indexed by the expectations that the representative agent holds about future inflation, $\eta_{t}$. Equivalently, the solution to the univariate model is indeterminate: the Blanchard-Kahn solution is not satisfied since there is no explosive root to match the number of expectational variables, that is $\pi_{t}$.

From a methodological and computational perspective, the latter case constitutes a challenge. Standard software packages such as Dynare do not allow for indeterminacy. Of course, a researcher could in principle code an estimation algorithm herself, following the methods outlined in Lubik and Schorfheide (2004). However, this approach requires a substantial amount of time and technical skills. Hence, the result is that in practice most of the papers simply rule out the possibility of indeterminacy, even if the model at hand could in principle allow for such a feature. From a purely technical point of view, the problem that a researcher faces when solving a LRE model such as the one presented in (4) using standard solution algorithms is the following. Under determinacy, the model already has a sufficient number of unstable roots to match the number of expectational variables. However, under indeterminacy, the model is missing one explosive root since it still has one expectational variable, but no unstable root. Therefore, our approach proposes to augment the original model by appending an independent process which could be either stable or unstable. The key insight consists of choosing this auxiliary process in a way to deliver the correct solution. When the original model is determinate, the auxiliary process must be stationary so that also the augmented representation satisfies the BlanchardKahn condition. In this case, the auxiliary process represents a separate block that does not affect the law of motion of the model variables. When the model is indeterminate, the additional process should however be explosive so that the Blanchard-Kahn condition is satisfied for the augmented system, even if not for the original model. In what follows, we apply this intuition to the example considered in this section and explain how to choose the auxiliary process.

Our methodology proposes to solve an augmented system of equations which can be dealt with by using standard solution algorithms such as Sims (2001) under both determinacy and indeterminacy. Consider the following augmented system

$$
\left\{\begin{array}{c}
E_{t}\left(\pi_{t+1}\right)=\phi_{\pi} \pi_{t}-r_{t}, \\
\omega_{t}=\left(\frac{1}{\alpha}\right) \omega_{t-1}-\nu_{t}+\eta_{t},
\end{array}\right.
$$

where $\omega_{t}$ is an independent autoregressive process, $\alpha \in[0,2]$ and $\nu_{t}$ is a newly defined mean- 


\begin{tabular}{lcc}
\hline \hline \multicolumn{3}{c}{ Blanchard-Kahn condition in the augmented representation } \\
\hline \hline Unstable Roots & $\begin{array}{c}\text { B-K condition in } \\
\text { augmented model }(8)\end{array}$ & Solution \\
\hline
\end{tabular}

Determinacy $\phi_{\pi}>1$

in original model (4)

\begin{tabular}{|c|c|c|c|}
\hline$\frac{1}{\alpha}<1$ & 1 & Satisfied & $\left.\pi_{t}=\frac{1}{\phi_{\pi}} r_{t}, \omega_{t}=\alpha \omega_{t-1}-\nu_{t}+\varepsilon_{t}\right\}$ \\
\hline$\frac{1}{\alpha}>1$ & 2 & Not satisfied & - \\
\hline
\end{tabular}

Indeterminacy $\phi_{\pi} \leq 1$

in original model (4)

\begin{tabular}{lccc}
\hline$\frac{1}{\alpha}<1$ & 0 & Not satisfied & - \\
$\frac{1}{\alpha}>1$ & 1 & Satisfied & $\left\{\pi_{t}=\phi_{\pi} \pi_{t-1}-r_{t-1}+\eta_{t}, \omega_{t}=0\right\}$ \\
\hline \hline
\end{tabular}

Table 1: The table reports the regions of the parameter space for which the Blanchard-Kahn condition in the augmented representation is satisfied, even when the original model is indeterminate.

zero sunspot shock with standard deviation $\sigma_{v}$. Table 1 summarizes the intuition behind our approach.

When the original LRE model in (4) is determinate, $\phi_{\pi}>1$, the Blanchard-Kahn condition for the augmented representation in (8) is satisfied when $1 / \alpha<1$. Indeed, for $\phi_{\pi}>1$ the original model has the same number of unstable roots as the number of expectational variables. Our methodology thus suggests to append a stable autoregressive process. When $\phi_{\pi}>1$ and $1 / \alpha<1$, the solution method of Sims (2001) delivers the same solution for the endogenous variable $\pi_{t}$ as in equation (5). Since the coefficient $1 / \alpha$ is smaller than 1 , the solution also includes the autoregressive process $\omega_{t}$. Importantly, its dynamics do not impact the endogenous variable $y_{t}$.

Considering the case of indeterminacy (i.e. $\phi_{\pi} \leq 1$ ), the original model has one expectational variable, but no unstable root, thus violating the Blanchard-Kahn condition. By appending an explosive autoregressive process, the augmented representation that we propose satisfies the Blanchard-Kahn condition and delivers the same solution as the one resulting from the methodology of Lubik and Schorfheide (2003) or Farmer et al. (2015) described by equation (7). Moreover, stability imposes conditions such that $\omega_{t}$ is always equal to zero, and the solution for the endogenous variable does not depend on the appended autoregressive process.

Summarizing, the choice of the coefficient $\frac{1}{\alpha}$ should be made as follows. For values of $\phi_{\pi}$ greater than 1, the Blanchard-Kahn condition for the augmented representation is satisfied for values of $\alpha$ greater than 1. Conversely, under indeterminacy (i.e. $\phi_{\pi} \leq 1$ ) the condition is satisfied 
when $\alpha$ is smaller than 1 . The choice of parametrizing the auxiliary process with $1 / \alpha$ instead of $\alpha$ induces a positive correlation between $\phi_{\pi}$ and $\alpha$ that facilitates the implementation of our method when estimating a model.

Finally, note that under both determinacy and indeterminacy, the exact value of $1 / \alpha$ is irrelevant for the law of motion of $\pi_{t}$. Under determinacy, the auxiliary process $\omega_{t}$ is stationary, but its evolution does not affect the law of motion of the model variables. Under indeterminacy, $\omega_{t}$ is always equal to zero. This makes clear that introducing the auxiliary processes does not affect the properties of the solution in the two cases. These processes only serve the purpose of providing the necessary explosive roots under indeterminacy and of creating the mapping between the sunspot shocks and the expectation errors. As we will see in Section 3, this result can be generalized and applies to more complicated models with potentially multiple degrees of indeterminacy.

\subsection{Choosing $\alpha$}

A natural question that arises with the approach we propose is how to choose $\alpha$. We consider the following three cases: (1) The researcher knows the analytic condition defining the region of determinacy; (2) she only has an relatively good idea of the threshold of the determinacy region; (3) the region of determinacy is completely unknown to the researcher. We consider the three cases separately.

We first consider the case in which the researcher is able to analytically derive the condition which defines when the model is determinate or indeterminate. For the example considered in this section, this case corresponds to knowing that when $\phi_{\pi} \leq 1$ the model in (4) is indeterminate. We thus suggest to write the parameter $\alpha$ as a function of the parameter $\phi_{\pi}$ so that the augmented representation in (8) always satisfies the Blanchard-Kahn condition. This can be obtained by setting $\alpha \equiv \phi_{\pi}$. When the original model is determinate $\left(\phi_{\pi}>1\right)$, the appended autoregressive process is stationary since $1 / \alpha<1$. Under indeterminacy $\left(\phi_{\pi} \leq 1\right)$ of the original model, the coefficient $1 / \alpha$ is greater than 1 and the appended process is therefore explosive. Hence, when the region of determinacy is known, the researcher should carefully choose $\alpha$ such that the augmented representation always delivers a solution under both determinacy and indeterminacy. Using the NK model in Lubik and Schorfheide (2004), we implement this suggestion in Section 5.1 where we estimate the model assuming that the researcher knows the region of determinacy.

There are however instances in which the researcher does not know the exact properties of the determinacy region. Suppose that the researcher does not know the region, but for values of the parameter $\phi_{\pi}$ slightly above 1 she can solve the original model under determinacy, while for values just below 1 the model is indeterminate. She thus has a relatively good idea that the 
threshold for determinacy is somewhere around 1, even if she is not able to derive the analytical condition. In this case, she could set a prior distribution for the parameter $\alpha$ such that there is a higher probability of drawing values above 1 when the parameter $\phi_{\pi}$ is greater than 1 and vice versa. Similarly, the variance-covariance matrix used by the Metropolis-Hastings algorithm to propose new draws should be chosen to display a positive correlation between the values of $\phi_{\pi}$ and $\alpha$. This practice would increase the likelihood of obtaining a solution in the augmented representation and therefore the efficiency of the algorithm.

Finally, it could be the case that the region of determinacy is completely unknown to the researcher. For a given draw of the parameter $\phi_{\pi}$, the researcher would like to make draws of $\alpha$ smaller or greater than 1 with equal probabilities. In this case, the researcher could use a uniform distribution over the interval $[0,2]$ or any symmetric interval around 1 as a prior distribution. Also, note that the prior distribution does not necessarily have to be continuous. A discrete probability distribution that allows to make draws of $\alpha$ to be either equal 0.5 or 1.5 could also be specified as a prior. In this context, the efficiency of the algorithm would also be improved if it were to be designed as follows. If for a given draw of $\phi_{\pi}$ and $\alpha$ the augmented representation in (8) does not have a solution, the algorithm should be coded as to make a new draw of $\alpha^{\prime}$ equal to the inverse of the earlier draw $\alpha$.

\section{$3 \quad$ Methodology}

Given the general class of LRE models described in Sims (2001), this paper proposes an augmented representation which embeds the solution for the model under both determinacy and indeterminacy. ${ }^{3}$ In particular, the augmented representation of the LRE model delivers solutions which under determinacy are equivalent to those obtained using standard solution algorithms, and under indeterminacy to those obtained using the methodology provided by Lubik and Schorfheide $(2003,2004)$ or equivalently Farmer et al. (2015). In the following, we generalize the intuition built in the previous section. Consider the following LRE model

$$
\Gamma_{0}(\theta) X_{t}=\Gamma_{1}(\theta) X_{t-1}+\Psi(\theta) \varepsilon_{t}+\Pi(\theta) \eta_{t}
$$

where $X_{t} \in R^{k}$ is a vector of endogenous variables, $\varepsilon_{t} \in R^{\ell}$ is a vector of exogenous shocks, $\eta_{t} \in R^{p}$ collects the one-step ahead forecast errors for the expectational variables of the system and $\theta \in \Theta$ is a vector of parameters. The matrices $\Gamma_{0}$ and $\Gamma_{1}$ are of dimension $k \times k$, possibly singular, and the matrices $\Psi$ and $\Pi$ are of dimension $k \times \ell$ and $k \times p$, respectively. Also, we

\footnotetext{
${ }^{3}$ In this paper we focus on linear rational expectation models. Schmitt-Grohé and Uribe (2004) and Judd (1998) use perturbation methods to solve DSGE models using higher order approximations.
} 
assume

$$
E_{t-1}\left(\varepsilon_{t}\right)=0, \quad \text { and } \quad E_{t-1}\left(\eta_{t}\right)=0
$$

We also define the $\ell \times \ell$ matrix $\Omega_{\varepsilon \varepsilon}$,

$$
\Omega_{\varepsilon \varepsilon} \equiv E_{t-1}\left(\varepsilon_{t} \varepsilon_{t}^{T}\right)
$$

which represents the covariance matrix of the exogenous shocks.

Consider a model whose maximum degree of indeterminacy is denoted by $m .{ }^{4}$ The proposed methodology appends to the original LRE model in (9) the following system of $m$ equations

$$
\omega_{t}=\Phi \omega_{t-1}+\nu_{t}-\eta_{f, t}, \quad \Phi \equiv\left[\begin{array}{ccc}
\frac{1}{\alpha_{1}} & & \mathbf{0} \\
& \ddots & \\
\mathbf{0} & & \frac{1}{\alpha_{m}}
\end{array}\right]
$$

where the vector $\eta_{f, t}$ is a subset of the endogenous shocks and the vectors $\left\{\omega_{t}, \nu_{t}, \eta_{f, t}\right\}$ are of dimension $m \times 1$. The equations in (12) are autoregressive processes whose innovations are linear combinations of a vector of sunspot shocks, $\nu_{t}$, and a subset of forecast errors, $\eta_{f, t}$, where $E_{t-1}\left(\nu_{t}\right)=E_{t-1}\left(\eta_{f, t}\right)=0$. As we will show below, the choice of which expectational errors to include in (12) does not affect the solution.

The intuition behind the proposed methodology works as in the example considered in the previous section. Let $m^{*}(\theta)$ denote the actual degree of indeterminacy associated with the parameter vector $\theta$. Under indeterminacy the Blanchard-Kahn condition for the original LRE model in (9) is not satisfied. Suppose that the system is characterized by $m^{*}(\theta)$ degrees of indeterminacy, then it is necessary to introduce $m^{*}(\theta)$ explosive roots to solve the model using standard solution algorithms. In this case, the (absolute value of the) $m^{*}(\theta)$ of the diagonal elements of the matrix $\Phi$ are assumed to be outside the unit circle, and the augmented representation is therefore determinate since the Blanchard-Kahn condition is now satisfied. On the other hand, under determinacy the (absolute value of the) diagonal elements of the matrix $\Phi$ are assumed to be all inside the unit circle, since the number of explosive roots of the original LRE model in (9) already equals the number of expectational variables in the model $\left(m^{*}(\theta)=0\right)$. Also, in this case the augmented representation is determinate due to the stability of the appended auxiliary processes. Importantly, as shown for the univariate example in Section 2, the block structure of the proposed methodology guarantees that the autoregressive process, $\omega_{t}$, does not affect the

\footnotetext{
${ }^{4}$ Denoting by $n$ the minimum number of unstable roots of a LRE model, the maximum degrees of indeterminacy are defined as $m \equiv p-n$. When the minimum number of unstable roots of a model is unknown, then $m$ coincides with number of expectational variables $p$. This represents the maximum degree of indeterminacy in any model with $p$ expectational variables.
} 
solution for the endogenous variables, $X_{t}$.

Denoting the newly defined vector of endogenous variables $\hat{X}_{t} \equiv\left(X_{t}, \omega_{t}\right)^{T}$ and the newly defined vector of exogenous shocks $\hat{\varepsilon}_{t} \equiv\left(\varepsilon_{t}, \nu_{t}\right)^{T}$, the system in (9) and (12) can be written as

$$
\hat{\Gamma}_{0} \hat{X}_{t}=\hat{\Gamma}_{1} \hat{X}_{t-1}+\hat{\Psi} \hat{\varepsilon}_{t}+\hat{\Pi} \eta_{t}
$$

where

$$
\hat{\Gamma}_{0} \equiv\left[\begin{array}{cc}
\Gamma_{0}(\theta) & \mathbf{0} \\
\mathbf{0} & \mathbf{I}
\end{array}\right], \quad \hat{\Gamma}_{1} \equiv\left[\begin{array}{cc}
\Gamma_{1}(\theta) & \mathbf{0} \\
\mathbf{0} & \Phi
\end{array}\right], \quad \hat{\Psi} \equiv\left[\begin{array}{cc}
\Psi(\theta) & \mathbf{0} \\
\mathbf{0} & \mathbf{I}
\end{array}\right], \quad \hat{\Pi} \equiv\left[\begin{array}{cc}
\Pi_{n}(\theta) & \Pi_{f}(\theta) \\
0 & -\mathbf{I}
\end{array}\right]
$$

and without loss of generality the matrix $\Pi$ in (9) is partitioned as $\Pi=\left[\begin{array}{lll}\Pi_{n} & \Pi_{f}\end{array}\right]$, where the matrices $\Pi_{n}$ and $\Pi_{f}$ are respectively of dimension $k \times(p-m)$ and $k \times m$.

Section 3.1 and 3.2 show that the augmented representation of the LRE model delivers solutions which under determinacy are equivalent to those obtained using standard solution algorithms, and under indeterminacy to those obtained using the methodology provided by Lubik and Schorfheide $(2003,2004)$ and Farmer et al. (2015). In order to simplify the exposition, when analyzing the case of indeterminacy we assume, without loss of generality, $m^{*}(\theta)=m$. As it will become clear, the case of $m^{*}(\theta)<m$ is a special case of what we present below.

\subsection{Equivalence under determinacy}

This section considers the case in which the original LRE is determinate, and shows the equivalence of the solution obtained using the proposed augmented representation with the one from the standard solution algorithm described in Sims (2001).

\subsubsection{Canonical solution}

Consider the LRE model in (9) and reported below as (14)

$$
\underset{k \times k k \times 1}{\Gamma_{0} X_{t}}=\underset{k \times k}{\Gamma_{1}} X_{t-1}+\underset{k \times 1}{\Psi} \varepsilon_{t}+\underset{k \times p_{p \times 1}}{\prod} \eta_{t}
$$

The method described in Sims (2001) delivers a solution, if it exists, by following four steps. First, Sims (2001) shows how to write the model in the form

$$
S Z^{T} X_{t}=T Z^{T} X_{t-1}+Q \Psi \varepsilon_{t}+Q \Pi \eta_{t}
$$


where $\Gamma_{0}=Q^{T} S Z^{T}$ and $\Gamma_{1}=Q^{T} T Z^{T}$ is the QZ decomposition of $\left\{\Gamma_{0}, \Gamma_{1}\right\}$, and the $k \times k$ matrices $Q$ and $Z$ are orthonormal, upper triangular and possibly complex. Also, the diagonal elements of $S$ and $T$ contain the generalized eigenvalues of $\left\{\Gamma_{0}, \Gamma_{1}\right\}$.

Second, since the QZ decomposition is not unique, Sims' algorithm chooses a decomposition that orders the equations so that the absolute values of the ratios of the generalized eigenvalues are placed in an increasing order, that is

$$
\left|t_{j j}\right| /\left|s_{j j}\right| \geq\left|t_{i i}\right| /\left|s_{i i}\right| \quad \text { for } j>i \text {. }
$$

The algorithm then partitions the matrices $S, T, Q$ and $Z$ as

$$
S=\left[\begin{array}{cc}
S_{11} & S_{12} \\
0 & S_{22}
\end{array}\right], \quad T=\left[\begin{array}{cc}
T_{11} & T_{12} \\
0 & T_{22}
\end{array}\right], \quad Z^{\prime}=\left[\begin{array}{c}
Z_{1} \\
Z_{2}
\end{array}\right], \quad Q=\left[\begin{array}{l}
Q_{1} \\
Q_{2}
\end{array}\right]
$$

where the first block corresponds to the system of equations for which $\left|t_{j j}\right| /\left|s_{j j}\right|<1$ and the second block groups the equations which are characterized by explosive roots, $\left|t_{j j}\right| /\left|s_{j j}\right|>1$.

The third step imposes conditions on the second, explosive block to guarantee the existence of at least one bounded solution. Defining the transformed variables

$$
\xi_{t} \equiv Z^{T} X_{t}=\left[\begin{array}{c}
\xi_{1, t} \\
(k-n) \times 1 \\
\xi_{2, t} \\
n \times 1
\end{array}\right],
$$

where $n$ is the number of explosive roots, and the transformed parameters

$$
\widetilde{\Psi} \equiv Q^{T} \Psi, \quad \text { and } \quad \widetilde{\Pi} \equiv Q^{T} \Pi
$$

the second block can be written as

$$
\xi_{2, t}=S_{22}^{-1} T_{22} \xi_{2, t-1}+S_{22}^{-1}\left(\widetilde{\Psi}_{2} \varepsilon_{t}+\widetilde{\Pi}_{2} \eta_{t}\right)
$$

Since this system of equations contains the explosive roots of the system, then a bounded solution, if it exists, will set

$$
\begin{aligned}
\xi_{2,0} & =0 \\
n \times 1 & =0, \\
\underset{n \times \ell \ell \times 1}{\tilde{\Psi}_{2} \varepsilon_{t}}+\underset{n \times p}{\tilde{\Pi}_{2} \eta_{t}} & =0,
\end{aligned}
$$


where $n$ also denotes the number of equations in $(21) .^{5}$

A necessary condition for the existence of a solution requires that the number of unstable roots $(n)$ equals the number of expectational variables $(p)$. In this section, we are considering the solution under determinacy and this guarantees that there are no degrees of indeterminacy $m^{*}(\theta)=0$. The sufficient condition then requires that the columns of the matrix $\tilde{\Pi}_{2}$ are linearly independent so that there is at least one bounded solution. In that case, the matrix $\tilde{\Pi}_{2}$ is a square, non-singular matrix and equation (21) imposes linear restrictions on the forecast errors, $\eta_{t}$, as a function of the fundamental shocks, $\varepsilon_{t}$,

$$
\eta_{t}=-\tilde{\Pi}_{2}^{-1} \tilde{\Psi}_{2} \varepsilon_{t}
$$

The fourth and last step finds the solution for the endogenous variables, $X_{t}$, by combining the restrictions in (20) and (22) with the system of stable equations in the first block,

$$
\begin{aligned}
\xi_{1, t} & =S_{11}^{-1} T_{11} \xi_{1, t-1}+S_{11}^{-1}\left(\widetilde{\Psi}_{1} \varepsilon_{t}+\widetilde{\Pi}_{1} \eta_{t}\right) \\
& =S_{11}^{-1} T_{11} \xi_{1, t-1}+S_{11}^{-1}\left(\widetilde{\Psi}_{1}-\widetilde{\Pi}_{1} \tilde{\Pi}_{2}^{-1} \tilde{\Psi}_{2}\right) \varepsilon_{t}
\end{aligned}
$$

Using the algorithm by Sims (2001), the solution of the LRE model in (14) is described by equations (20), (22), and (23).

\subsubsection{Augmented representation}

We now consider the methodology proposed in this paper, and we augment the LRE model in (14) with the following system of $m$ equations

$$
\omega_{t}=\Phi \omega_{t-1}+\nu_{t}-\eta_{f, t}, \quad \Phi \equiv\left[\begin{array}{ccc}
\frac{1}{\alpha_{1}} & & \mathbf{0} \\
& \ddots & \\
\mathbf{0} & & \frac{1}{\alpha_{m}}
\end{array}\right]
$$

where $\Phi$ is a $m \times m$ diagonal matrix. Since the original model in (14) is determinate, then we assume that all the diagonal elements $\alpha_{i}$ belong to the interval [1,2]. Therefore, we are appending a system of stable equations, and we show that the solution for the endogenous variables, $X_{t}$, is equivalent to the one found in Section 3.1.1. Defining the augmented vector of endogenous variables, $\hat{X}_{t} \equiv\left(X_{t}, \omega_{t}\right)^{T}$ and the augmented vector of exogenous shocks $\hat{\varepsilon}_{t} \equiv\left(\varepsilon_{t}, \nu_{t}\right)^{T}$, the

\footnotetext{
${ }^{5}$ Note that the eigenvalues of $S_{22}^{-1} T_{22}$ are all greater than one in absolute value.
} 
representation that we propose takes the form

$$
\hat{\Gamma}_{0} \hat{X}_{t}=\hat{\Gamma}_{1} \hat{X}_{t-1}+\hat{\Psi} \hat{\varepsilon}_{t}+\hat{\Pi} \eta_{t}
$$

where

$$
\hat{\Gamma}_{0} \equiv\left[\begin{array}{cc}
\Gamma_{0} & \mathbf{0} \\
\mathbf{0} & \mathbf{I}
\end{array}\right], \quad \hat{\Gamma}_{1} \equiv\left[\begin{array}{cc}
\Gamma_{1} & \mathbf{0} \\
\mathbf{0} & \Phi
\end{array}\right], \quad \hat{\Psi} \equiv\left[\begin{array}{cc}
\Psi & \mathbf{0} \\
\mathbf{0} & \mathbf{I}
\end{array}\right], \quad \hat{\Pi} \equiv\left[\begin{array}{cc}
\Pi_{n} & \Pi_{f} \\
0 & -\mathbf{I}
\end{array}\right]
$$

and without loss of generality the matrix $\Pi$ is partitioned as $\Pi=\left[\begin{array}{ll}\Pi_{n} & \Pi_{f}\end{array}\right]$, where the matrices $\Pi_{n}$ and $\Pi_{f}$ are respectively of dimension $k \times(p-m)$ and $k \times m$.

We can find a solution to the augmented representation in (24) by using Sims' algorithm. Similarly to the previous section, we follow the four steps which describe the algorithm. First, the solution algorithm performs the QZ decomposition of the matrices $\left\{\hat{\Gamma}_{0}, \hat{\Gamma}_{1}\right\}$ and the augmented representation takes the form

$$
\hat{S} \hat{Z}^{T} \hat{X}_{t}=\hat{T} \hat{Z}^{T} \hat{X}_{t-1}+\hat{Q} \hat{\Psi} \hat{\varepsilon}_{t}+\hat{Q} \hat{\Pi} \eta_{t}
$$

where $\hat{\Gamma}_{0}=\hat{Q}^{T} \hat{S} \hat{Z}^{T}$ and $\hat{\Gamma}_{1}=\hat{Q}^{T} \hat{T} \hat{Z}^{T}$ is the QZ decomposition of $\left\{\hat{\Gamma}_{0}, \hat{\Gamma}_{1}\right\}$, and

$$
\hat{S}=\left[\begin{array}{ccc}
S_{11} & \mathbf{0} & S_{12} \\
\mathbf{0} & \mathbf{I} & \mathbf{0} \\
\mathbf{0} & \mathbf{0} & S_{22}
\end{array}\right], \quad \hat{T}=\left[\begin{array}{ccc}
T_{11} & \mathbf{0} & T_{12} \\
\mathbf{0} & \Phi & \mathbf{0} \\
\mathbf{0} & \mathbf{0} & T_{22}
\end{array}\right], \quad \hat{Z}^{T}=\left[\begin{array}{cc}
Z_{1} & \mathbf{0} \\
\mathbf{0} & \mathbf{I} \\
Z_{2} & \mathbf{0}
\end{array}\right], \quad \hat{Q}=\left[\begin{array}{cc}
Q_{1} & \mathbf{0} \\
\mathbf{0} & \mathbf{I} \\
Q_{2} & \mathbf{0}
\end{array}\right]
$$

Importantly, note that the inner matrices of $\left\{\hat{S}, \hat{T}, \hat{Z}^{T}, \hat{Q}\right\}$ are the same as those which define the matrices $\left\{S, T, Z^{T}, Q\right\}$ in (16) found in the previous section.

Second, the algorithm chooses a QZ decomposition which groups the equations in a stable and an explosive block. Since this section assumes that the original model is determinate and that the diagonal elements of the matrix $\Phi$ are within the unit circle, the explosive block corresponds to the third system of equations in (25) which is characterized by explosive roots. Recalling the definition of the matrices $\hat{\Psi}$ and $\hat{\Pi}$, the system of equations in the third block is

$$
\xi_{2, t}=S_{22}^{-1} T_{22} \xi_{2, t-1}+S_{22}^{-1}\left(\widetilde{\Psi}_{2} \varepsilon_{t}+\widetilde{\Pi}_{2} \eta_{t}\right)
$$

The third step imposes conditions to guarantee the existence of a bounded solution. However, the explosive block in (26) is identical to the system of equations found in (19). Therefore, the algorithm imposes the same restrictions to guarantee the existence of a bounded solution, that 


$$
\xi_{2,0}=0
$$

and as found earlier

$$
\eta_{t}=-\widetilde{\Pi}_{2}^{-1} \widetilde{\Psi}_{2} \varepsilon_{t}
$$

Finally, the last step combines these restrictions with the system of equations in the stable block which corresponds to the first and second systems of equations in (25),

$$
\begin{aligned}
\xi_{1, t} & =S_{11}^{-1} T_{11} \xi_{1, t-1}+S_{11}^{-1}\left(\widetilde{\Psi}_{1}-\widetilde{\Pi}_{1} \tilde{\Pi}_{2}^{-1} \tilde{\Psi}_{2}\right) \varepsilon_{t} \\
\omega_{t} & =\Phi \omega_{t-1}+\nu_{t}-\eta_{f, t} .
\end{aligned}
$$

The solution in $(27) \sim(30)$ obtained for the augmented representation of the LRE model delivers the same solution for the endogenous variables of interest, $X_{t}$, found in the previous section and defined in equations (20), (22), and (23).

Two remarks should be made when comparing the two solutions. First, as shown in (28), the forecast errors are only a function of the exogenous shocks $\varepsilon_{t}$, and not of the sunspot shocks $v_{t}$. It is therefore clear that the endogenous variables, $X_{t}$, of the original LRE model in (14) do not respond to sunspot shocks either, as expected under determinacy. Second, (29) and (30) indicate that under determinacy the appended system of equations constitutes a separate block, which does not affect the dynamics of the endogenous variables, $X_{t}$, and therefore the likelihood function associated with $X_{t}$.

\subsection{Equivalence under indeterminacy}

This section shows the equivalence of the solutions obtained for a LRE model under indeterminacy using the proposed augmented representation and the methodology of Lubik and Schorfheide (2003, 2004).

\subsubsection{Lubik and Schorfheide (2003)}

As in Section 3.1, we consider the LRE model in (14) and reported below as (31)

$$
\Gamma_{0} X_{t}=\Gamma_{1} X_{t-1}+\Psi \varepsilon_{t}+\Pi \eta_{t}
$$

In this section we assume that the model is indeterminate, and we present the method used by Lubik and Schorfheide (2003). The authors implement the first two steps of the algorithm by Sims 
(2001) and described in Section 3.1.1. ${ }^{6}$ They proceed by first applying the QZ decomposition to the LRE model in (31) and then ordering the resulting system of equations in a stable and an explosive block as defined in (15) and (16). However, their approach differs in the third step when the algorithm imposes restrictions to guarantee the existence of a bounded solution. In particular, the restrictions in (20) and (21) reported below as (32) and (33) require that

$$
\begin{aligned}
\xi_{2,0} & =0, \\
n \times 1 & =0 . \\
\underset{n \times \ell \ell \times 1}{\tilde{\Psi}_{2} \varepsilon_{t}}+\underset{n \times p}{\tilde{\Pi}_{2} \eta_{t}} & =0 .
\end{aligned}
$$

Nevertheless, it is clear that the system of equation in (33) is indeterminate since the number of forecast errors exceeds the number of explosive roots $(p>n)$. Equivalently, there are less equations $(n)$ than the number of variables to solve for $(p)$. To characterize the full set of solutions to equation (33), Lubik and Schorfheide (2003) decompose the matrix $\tilde{\Pi}_{2}$ using the following singular value decomposition

$$
\underset{n \times p}{\tilde{\Pi}_{2}} \equiv \underset{n \times n}{U}\left[\begin{array}{cc}
D_{11} & \mathbf{0} \\
n \times n & n \times m
\end{array}\right] \underset{p \times p}{V^{T}},
$$

where $m$ represents the degrees of indeterminacy. Given the partition $\underset{p \times p}{V} \equiv\left[\begin{array}{cc}V_{1} & V_{2} \\ p \times n & p \times m\end{array}\right]$, equation (33) can be written as

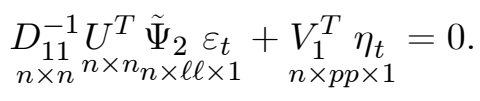

Since the system is indeterminate, Lubik and Schorfheide append additional $m$ equations,

$$
\underset{m \times \ell \ell \times 1}{\widetilde{M}} \varepsilon_{t}+\underset{m \times m m \times 1}{M_{\zeta}} \zeta_{t}=\underset{m \times p}{V_{2}^{T}} \eta_{t}
$$

The $m \times 1$ vector $\zeta_{t}$ is a set of sunspot shocks that is assumed to have mean zero, covariance matrix $\Omega_{\zeta \zeta}$ and to be uncorrelated with the fundamental shocks, $\varepsilon_{t}$, that is

$$
E\left[\zeta_{t}\right]=0, \quad E\left[\zeta_{t} \varepsilon_{t}^{T}\right]=0, \quad E\left[\zeta_{t} \zeta_{t}^{T}\right]=\Omega_{\zeta \zeta}
$$

The matrix $\widetilde{M}$ captures the correlation of the forecast errors, $\eta_{t}$, with fundamentals, $\varepsilon_{t}$, and Lubik and Schorfheide (2003) choose the normalization $M_{\zeta}=I_{m}$. Appending the system of

\footnotetext{
${ }^{6}$ It is relevant to mention that in this section the matrices obtained from the QZ decomposition and the ordering of the equations into a stable and an explosive block differ from those in Section 3.1 both in terms of the elements constituing them and their dimensions. However, we opted to use the same notation for simplicity.
} 
equations in (35) to the equations in (34), the expectational errors can be written as a function of the fundamental shocks, $\varepsilon_{t}$, and the sunspot shocks, $\zeta_{t}$,

$$
\underset{p \times 1}{\eta_{t}}=\left(\underset{p \times n}{-V_{1} D_{11}^{-1} U_{1}^{T} \tilde{\Psi}_{2}+\underset{n \times n n \times \ell}{V_{2}} \underset{m \times m}{\widetilde{M}}}\right) \underset{\ell \times 1}{\varepsilon_{t}}+\underset{p \times m}{V_{2}} \underset{m \times 1}{\zeta_{t}} .
$$

More compactly,

$$
\underset{p \times 1}{\eta_{t}}=\left(\underset{p \times n n \times \ell}{V_{1}}+\underset{p \times m}{V_{2}} \underset{m \times \ell}{\widetilde{M}}\right) \underset{\ell \times 1}{\varepsilon_{t}}+\underset{p \times m}{V_{2}} \underset{m \times 1}{\zeta_{t}}
$$

where

$$
\underset{n \times \ell}{N} \equiv \underset{n \times n}{-D_{11}^{-1} U_{n \times n n \times \ell}^{T} \tilde{\Psi}_{2}}
$$

is a function of the parameters of the model.

Given the earlier restriction in (32) and (36), the fourth step in the algorithm combines these equations with the system of stable equations in the first block as in Section 3.1.1,

$$
\begin{aligned}
\xi_{1, t} & =S_{11}^{-1} T_{11} \xi_{1, t-1}+S_{11}^{-1}\left(\widetilde{\Psi}_{1} \varepsilon_{t}+\widetilde{\Pi}_{1} \eta_{t}\right) \\
& =S_{11}^{-1} T_{11} \xi_{1, t-1}+S_{11}^{-1}\left(\widetilde{\Psi}_{1}+\widetilde{\Pi}_{1} V_{1} N+\widetilde{\Pi}_{1} V_{2} \widetilde{M}\right) \varepsilon_{t}+S_{11}^{-1}\left(\widetilde{\Pi}_{1} V_{2}\right) \zeta_{t} .
\end{aligned}
$$

Using the method in Lubik and Schorfheide (2003), the solution for the original LRE model under indeterminacy is described by (32), (36) and (37).

\subsubsection{Augmented representation}

We now consider the augmented representation as in (24) and reported below as

$$
\hat{\Gamma}_{0} \hat{X}_{t}=\hat{\Gamma}_{1} \hat{X}_{t-1}+\hat{\Psi} \hat{\varepsilon}_{t}+\hat{\Pi} \eta_{t}
$$

where $\hat{X}_{t} \equiv\left(X_{t}, \omega_{t}\right)^{T}, \hat{\varepsilon}_{t} \equiv\left(\varepsilon_{t}, \nu_{t}\right)^{T}$ and

$$
\hat{\Gamma}_{0} \equiv\left[\begin{array}{cc}
\Gamma_{0} & \mathbf{0} \\
\mathbf{0} & \mathbf{I}
\end{array}\right], \quad \hat{\Gamma}_{1} \equiv\left[\begin{array}{cc}
\Gamma_{1} & \mathbf{0} \\
\mathbf{0} & \Phi
\end{array}\right], \quad \hat{\Psi} \equiv\left[\begin{array}{cc}
\Psi & \mathbf{0} \\
\mathbf{0} & \mathbf{I}
\end{array}\right], \quad \hat{\Pi} \equiv\left[\begin{array}{cc}
\Pi_{n} & \Pi_{f} \\
0 & -\mathbf{I}
\end{array}\right] .
$$

where the matrix $\Pi$ is partitioned as $\Pi=\left[\begin{array}{ll}\Pi_{n} & \Pi_{f}\end{array}\right]$ without loss of generality.

The novelty of our approach is that, given our representation, we can easily obtain the solution by using Sims' algorithm even when the original LRE is assumed to be indeterminate. It is enough to assume that the auxiliary processes $\omega_{t}$ are characterized by explosive roots, or equivalently that the diagonal elements of the matrix $\Phi$ are outside the unit circle. This guarantees that the 
Blanchard-Kahn condition for the augmented representation is satisfied and, given the analytic form that we propose for the auxiliary processes, we show that the solution for the endogenous variables of interest, $X_{t}$, is equivalent to the method of Lubik and Schorfheide (2003).

To show this result, we simply apply the four steps of the algorithm described in Sims (2001) to the proposed augmented representation. First, the QZ decomposition of (38) takes the form

$$
\hat{S} \hat{Z}^{T} \hat{X}_{t}=\hat{T} \hat{Z}^{T} \hat{X}_{t-1}+\hat{Q} \hat{\Psi} \hat{\varepsilon}_{t}+\hat{Q} \hat{\Pi} \eta_{t}
$$

where $\hat{\Gamma}_{0}=\hat{Q}^{T} \hat{S} \hat{Z}^{T}$ and $\hat{\Gamma}_{1}=\hat{Q}^{T} \hat{T} \hat{Z}^{T}$ is the QZ decomposition ${ }^{7}$ of $\left\{\hat{\Gamma}_{0}, \hat{\Gamma}_{1}\right\}$ and

$$
\hat{S}=\left[\begin{array}{ccc}
S_{11} & S_{12} & \mathbf{0} \\
\mathbf{0} & S_{22} & \mathbf{0} \\
\mathbf{0} & \mathbf{0} & \mathbf{I}
\end{array}\right], \quad \hat{T}=\left[\begin{array}{ccc}
T_{11} & T_{12} & \mathbf{0} \\
\mathbf{0} & T_{22} & \mathbf{0} \\
\mathbf{0} & \mathbf{0} & \Phi
\end{array}\right], \quad \hat{Z}^{T}=\left[\begin{array}{cc}
Z_{1} & \mathbf{0} \\
Z_{2} & \mathbf{0} \\
\mathbf{0} & \mathbf{I}
\end{array}\right], \quad \hat{Q}=\left[\begin{array}{cc}
Q_{1} & \mathbf{0} \\
Q_{2} & \mathbf{0} \\
\mathbf{0} & \mathbf{I}
\end{array}\right]
$$

Second, the QZ decomposition chosen by the algorithm groups the explosive dynamics of the model in the second and third system of equations in (40), which are reported below as (42)

$$
\left[\begin{array}{cc}
S_{22} & \mathbf{0} \\
\mathbf{0} & \mathbf{I}
\end{array}\right]\left[\begin{array}{c}
\xi_{2} \\
\omega_{t}
\end{array}\right]=\left[\begin{array}{cc}
T_{22} & \mathbf{0} \\
\mathbf{0} & \Phi
\end{array}\right]\left[\begin{array}{c}
\xi_{2, t-1} \\
\omega_{t-1}
\end{array}\right]+\left[\begin{array}{cc}
Q_{2} & \mathbf{0} \\
\mathbf{0} & \mathbf{I}
\end{array}\right]\left(\hat{\Psi} \hat{\varepsilon}_{t}+\hat{\Pi} \eta_{t}\right)
$$

where $\xi_{t} \equiv Z^{T} X_{t}$.

In the third step, the following restrictions are imposed,

$$
\begin{aligned}
\xi_{2,0} & =0 \\
n \times 1 & \\
\omega_{0} & =0 \\
m \times 1 & \\
{\left[\begin{array}{cc}
Q_{2} & \mathbf{0} \\
\mathbf{0} & \mathbf{I}
\end{array}\right]\left(\hat{\Psi} \hat{\varepsilon}_{t}+\hat{\Pi} \eta_{t}\right) } & =0 .
\end{aligned}
$$

Recalling the definition of $\hat{\Psi}$ and $\hat{\Pi}$ and that we define $\widetilde{\Psi} \equiv Q^{T} \Psi$, and $\widetilde{\Pi} \equiv Q^{T} \Pi$, then equation (45) can be written as

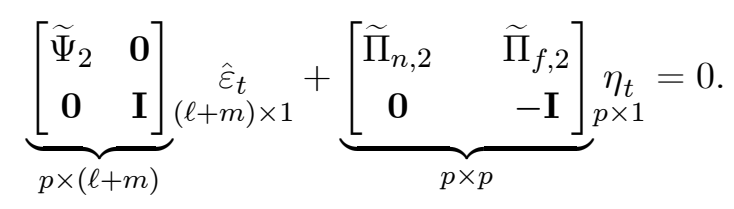

\footnotetext{
${ }^{7}$ Note that the inner matrices of $\left\{\hat{S}, \hat{T}, \hat{Z}^{T}, \hat{Q}\right\}$ are the same as those which define the matrices $\left\{S, T, Z^{T}, Q\right\}$ found from the QZ decomposition using the methodology of Lubik and Schorfheide (2003).
} 
Equation (46) shows transparently how the explosive auxiliary process that we append help to solve the original LRE model under indeterminacy. The system of equations in (46) is determinate as the number of equations defined by the explosive roots of the system equals the number of expectational errors of the model. This guarantees that the necessary condition for the existence of a bounded solution for the augmented representation is satisfied. Assuming that the columns of the matrix associated with the vector of non-fundamental shocks, $\eta_{t}$, are linearly independent, we can therefore impose linear restrictions on the forecast errors as a function of the augmented vector of exogenous shocks $\hat{\varepsilon}_{t} \equiv\left(\varepsilon_{t}, \nu_{t}\right)^{T}$,

$$
\eta_{t}=-\left[\begin{array}{cc}
\widetilde{\Pi}_{n, 2}^{-1} \widetilde{\Psi}_{2} & \widetilde{\Pi}_{n, 2}^{-1} \widetilde{\Pi}_{f, 2} \\
\mathbf{0} & -\mathbf{I}
\end{array}\right] \hat{\varepsilon}_{t}
$$

More compactly,

$$
\eta_{t}=C_{1} \varepsilon_{t}+C_{2} \nu_{t}
$$

where $C_{1} \equiv-\left[\begin{array}{c}\widetilde{\Pi}_{n, 2}^{-1} \widetilde{\Psi}_{2} \\ \mathbf{0}\end{array}\right]$ and $C_{2} \equiv-\left[\begin{array}{c}\widetilde{\Pi}_{n, 2}^{-1} \widetilde{\Pi}_{f, 2} \\ -\mathbf{I}\end{array}\right]$ are a function of the structural parameters of the model.

The last step of Sims' algorithm combines the restrictions in (43), (44) and (48) with the stationary block derived from the QZ decomposition in (40),

$$
\begin{aligned}
\xi_{1, t} & =S_{11}^{-1} T_{11} \xi_{1, t-1}+S_{11}^{-1}\left(\widetilde{\Psi}_{1} \varepsilon_{t}+\widetilde{\Pi}_{1} \eta_{t}\right) \\
& =S_{11}^{-1} T_{11} \xi_{1, t-1}+S_{11}^{-1}\left(\widetilde{\Psi}_{1}+\widetilde{\Pi}_{1} C_{1}\right) \varepsilon_{t}+S_{11}^{-1}\left(\widetilde{\Pi}_{1} C_{2}\right) v_{t}
\end{aligned}
$$

\subsubsection{Indeterminate equilibria and equivalent characterizations}

The indeterminate equilibria found using the methodology of Lubik and Schorfheide (2003) are parametrized by two sets of parameters. The first set is defined by $\theta_{1} \in \Theta_{1}$, where $\theta_{1} \equiv \operatorname{vec}\left(\Gamma_{0}, \Gamma_{1}, \Psi, \Omega_{\varepsilon \varepsilon}\right)^{T}$ is a vector of structural parameters of the model as well as the covariance matrix of the exogenous shocks. The second set corresponds to $\theta_{2} \in \Theta_{2}$, where $\theta_{2} \equiv \operatorname{vec}\left(\Omega_{\zeta \zeta}, \widetilde{M}\right)^{T}$ is a parameter vector related to the additional equations introduced in (35) and reported below as (50),

$$
\underset{m \times \ell \ell \times 1}{\widetilde{M}} \varepsilon_{t}+\underset{m \times m m \times 1}{M_{\zeta}} \zeta_{t}=\underset{m \times p}{V_{2 \times 1}^{T}} \eta_{t}
$$

Given the normalization $M_{\zeta}=I$ chosen by Lubik and Schorfheide (2004), equation (50) introduces $m \times(m+1) / 2$ parameters associated with the covariance matrix of the sunspot shocks, $\Omega_{\zeta \zeta}$, and additional $m \times \ell$ parameters of the matrix $\widetilde{M}$ that is related to the covariances between 
$\eta_{t}$ and $\varepsilon_{t}$. In Appendix A, we show how the normalization chosen in Lubik and Schorfheide (2004) maps into the methodology we propose.

The characterization of a Lubik-Schorfheide equilibrium is a vector $\theta^{L S} \in \Theta^{L S}$, where $\Theta^{L S}$ is defined as

$$
\Theta^{L S} \equiv\left\{\Theta_{1}, \Theta_{2}\right\}
$$

Similarly, the full characterization of the solutions under indeterminacy using the proposed augmented representation is parametrized by the set of parameters $\theta_{1} \in \Theta_{1}$ common between the two methodologies, and the set of additional parameters $\theta_{3} \in \Theta_{3}$, where $\theta_{3} \equiv \operatorname{vec}\left(\Omega_{\nu \nu}, \Omega_{\nu \varepsilon}\right)^{T}$. Using our approach, we also introduce $m \times(m+1) / 2$ parameters associated with the covariance matrix of the sunspot shocks, $\Omega_{v v}$, and $m \times \ell$ parameters of the covariances, $\Omega_{v \varepsilon}$, between the sunspot shock $v_{t}$ and the exogenous shocks $\varepsilon_{t}$. A Bianchi-Nicolò equilibrium is characterized by a parameter vector $\theta^{B N} \in \Theta^{B N}$, where $\Theta^{B N}$ is defined as

$$
\Theta^{B N} \equiv\left\{\Theta_{1}, \Theta_{3}\right\}
$$

The following theorem establishes the equivalence between the characterizations of indeterminate equilibria obtained by using the methodology in Lubik and Schorfheide (2003) and the proposed augmented representation.

Theorem 1 Let $\theta^{L S}$ and $\theta^{B N}$ be two alternative parametrizations of an indeterminate equilibrium of the model

$$
\Gamma_{0} X_{t}=\Gamma_{1} X_{t-1}+\Psi \varepsilon_{t}+\Pi \eta_{t}
$$

For every BN equilibrium, parametrized by $\theta^{B N}$, there is a unique matrix $\tilde{M}$ and a unique matrix $\Omega_{\zeta \zeta}$ such that $\theta_{2}=\operatorname{vec}\left(\Omega_{\zeta \zeta}, \tilde{M}\right)^{T}$, and $\left\{\theta_{1}, \theta_{2}\right\} \in \Theta^{L S}$ defines an equivalent $L S$ equilibrium. Conversely, for every $L S$ equilibrium, parametrized by $\theta^{L S}$, there is a unique matrix $\Omega_{v v}$ and a unique covariance matrix $\Omega_{v \varepsilon}$ such that $\theta_{3}=\operatorname{vec}\left(\Omega_{v v}, \Omega v \varepsilon\right)^{T}$, and $\left\{\theta_{1}, \theta_{3}\right\} \in \Theta^{B N}$ defines an equivalent BN equilibrium.

Proof. See Appendix B.

In the paper Farmer et al. (2015), the authors also show that their characterization of indeterminate equilibria is equivalent to Lubik and Schorfheide (2003). Therefore, the following corollary holds.

Corollary 2 Given a parametrization $\theta^{B N}$ of a Bianchi-Nicolò indeterminate equilibrium, there exists a unique mapping into the parametrization of an indeterminate equilibrium for Farmer et al. (2015), and vice-versa. 
Moreover, the following two considerations support Corollary 3 below, which describes a relevant result on the likelihood function of the augmented representation. First, as emphasized in this section, the reduced form of the augmented representation has a block structure which ensures that solution for endogenous variables in $X_{t}$ is not a function of the autoregressive processes, $\omega_{t}$. Second, note that the appended autoregressive processes, $\omega_{t}$, have no economic interpretation and therefore have no relation with the observable variables used in a measurement equation. These two considerations imply that the parameters of the matrix $\Phi$ introduced with the augmented representation are not identified (within a certain region). Corollary 3 then follows. ${ }^{8}$

Corollary 3 The likelihood function associated with the newly defined vector of endogenous variables, $\hat{X}_{t}$, does not depend on the additional parameters included in the augmented representation, $\Phi$, and is equivalent to the likelihood function associated with the endogenous variables, $X_{t}$.

While Section 3.1 shows that the augmented representation of the LRE model delivers solutions which under determinacy are equivalent to those obtained using standard solution algorithms, Theorem 1 proves that under indeterminacy the solutions of our methodology are equivalent to those obtained using Lubik and Schorfheide (2003, 2004) and Farmer et al. (2015). This theoretical result is crucial for the estimation exercises conducted in Section 5. The augmented representation guarantees that the Metropolis-Hastings algorithm explores the entire domain of the parameter space.

\section{Analytic example}

This section considers the canonical NK model to provide an analytic example of the theoretical result presented in Section 3. Let

$$
\begin{aligned}
x_{t} & =E_{t}\left(x_{t+1}\right)-\tau\left(R_{t}-E_{t}\left(x_{t+1}\right)\right) \\
\pi_{t} & =\beta E_{t-1}\left(\pi_{t+1}\right)+\kappa x_{t} \\
R_{t} & =\psi \pi_{t}+\varepsilon_{R, t} \\
\eta_{1, t} & =x_{t}-E_{t-1}\left(x_{t}\right) \\
\eta_{2, t} & =\pi_{t}-E_{t-1}\left(\pi_{t}\right)
\end{aligned}
$$

\footnotetext{
${ }^{8}$ Notice that Corollary 3 holds when the augmented representation has a unique solution. This happens in two cases. First, values of the structural parameters $\theta$ which guarantee determinacy in the original LRE model should be combined with values for $\alpha_{i}$ in the matrix $\Phi$ whose absolute value lies within the unit circle. Second, values of the structural parameters $\theta$ for which the original model is indeterminate should be combined with (absolute) values of $\alpha_{i}$ outside the unit circle.
} 
where equations $(53) \sim(55)$ represent the dynamic IS curve, the NK Phillips curve and a monetary policy reaction function, respectively. The variable $x_{t}$ represents log deviations of GDP from a trend path and $\pi_{t}$ and $R_{t}$ are log deviations from the steady state level of inflation and the nominal interest rate. The one-step ahead forecast errors for the deviations of output from its trend and of inflation from its steady state are defined in (56) and (57). This model can be expressed in matrix form as

$$
\Gamma_{0} X_{t}=\Gamma_{1} X_{t-1}+\Psi \varepsilon_{t}+\Pi \eta_{t}
$$

where $X_{t}=\left(x_{t}, \pi_{t}, E_{t}\left(x_{t+1}\right), E_{t}\left(\pi_{t+1}\right)\right)^{T}, \varepsilon_{t}=\left(\varepsilon_{R, t}\right)$ and $\eta_{t}=\left(\eta_{1, t}, \eta_{2, t}\right)^{T}$.

It is well known that the region of determinacy is associated with an active response of the monetary authority to changes in inflation, a condition satisfied when $|\psi|>1$. Alternatively, the equilibrium is indeterminate when the monetary policy is "passive", that is $0<|\psi| \leq 1$. In the latter case, there is one degree of indeterminacy $(m=1)$ since there are two forecast errors while the system is characterized by only one unstable root. ${ }^{9}$ Given that $m=1$, the proposed methodology consists in appending to the original LRE model in (58) the following equation

$$
\omega_{t}=\frac{1}{\alpha} \omega_{t-1}+\nu_{t}-\eta_{2, t}
$$

To provide the intuition, consider $\alpha \equiv|\psi|$. When the monetary authority is "passive," indeterminacy arises and the Blanchard-Kahn condition for the original LRE model is not satisfied. Our representation augments the system in (58) with the explosive autoregressive process in (59). Note that under indeterminacy $0<|\psi| \leq 1$, which implies $1 / \alpha>1$. The augmented representation not only mechanically satisfies the Blanchard-Kahn condition, but, as proven in Theorem 1, it describes all the set of equilibria which would be equivalently obtained using the methodology of Lubik and Schorfheide (2003, 2004) or Farmer et al. (2015). Alternatively, when the monetary policy adopts an "active" stance, the original system is determinate and the auxiliary autoregressive process is stationary (i.e. $0<1 / \alpha<1$ ), thus satisfying the Blanchard-Kahn condition under determinacy. Importantly, as shown both in this example and more generally in Section 3, the block structure of the augmented representation ensures that the endogenous variables contained in the vector $X_{t}$ are not a function of the process $\omega_{t}$ for both regions of the parameter space.

We now show that the equivalence results in Section 3 hold for the NK model described by $(53) \sim(57)$. As described in the previous section, the proposed methodology defines a new

\footnotetext{
${ }^{9}$ As shown in Appendix C, one of the roots of the system is always outside the unit circle. This implies that the maximum degree of indeterminacy is $m=1$, and that we append only one auxiliary autoregressive process. However, it is not always possible to derive this analytical results. Under those circumstances, the number of auxiliary processes to append to the original LRE model equals the number of expectational variable. The latter indeed corresponds to the maximum degree of indeterminacy of the original model.
} 
vector of endogenous variables $\hat{X}_{t} \equiv\left(X_{t}, \omega_{t}\right)^{T}=\left(x_{t}, \pi_{t}, E_{t}\left(x_{t+1}\right), E_{t}\left(\pi_{t+1}\right), \omega_{t}\right)^{T}$ and a newly defined vector of exogenous shocks as $\hat{\varepsilon}_{t} \equiv\left(\varepsilon_{t}, \nu_{t}\right)^{T}=\left(\varepsilon_{R, t}, \nu_{t}\right)^{T}$. The system in (58) and (59) can then be written as

$$
\hat{\Gamma}_{0} \hat{X}_{t}=\hat{\Gamma}_{1} \hat{X}_{t-1}+\hat{\Psi} \hat{\varepsilon}_{t}+\hat{\Pi} \eta_{t}
$$

The representation in (60) always delivers a solution of the following form under both determinacy and indeterminacy,

$$
\hat{X}_{t}=\hat{T} \hat{X}_{t-1}+\hat{R} \hat{\varepsilon}_{t}
$$

In what follows, we show the equivalence of our solutions under determinacy with the one from Sims (2001), and under indeterminacy with the solution proposed by Farmer et al. (2015).

\subsection{Determinacy}

This section clarifies the details for the equivalence of the solutions which are obtained in the determinacy region of the parameter space when using the following two representations:

a) The matrix representation of the model in (58) and reported here as equation (62)

$$
\Gamma_{0} X_{t}=\Gamma_{1} X_{t-1}+\Psi \varepsilon_{t}+\Pi \eta_{t}
$$

b) The proposed augmented representation in (60) and reported here as equation (63)

$$
\hat{\Gamma}_{0} \hat{X}_{t}=\hat{\Gamma}_{1} \hat{X}_{t-1}+\hat{\Psi} \hat{\varepsilon}_{t}+\hat{\Pi} \eta_{t}
$$

Representations a) and b) deliver the equilibrium conditions reported in Table 2 , where $\alpha \equiv$ $|\psi|>1 .{ }^{10}$ Comparing the obtained solutions, it is clear that they are equivalent. While our augmented representation potentially allows for the sunspot shock to affect the model dynamics, the coefficients which determine its impact on the endogenous variables equal zero. Moreover, the dynamics of the endogenous variables $X_{t}=\left(x_{t}, \pi_{t}, E_{t}\left(x_{t+1}\right), E_{t}\left(\pi_{t+1}\right)\right)^{\prime}$ are not affected by the autoregressive process $\omega_{t}$ since it constitutes a separate block.

\subsection{Indeterminacy}

Under indeterminacy, the Blanchard-Kahn condition is not satisfied and to solve the model we use the solution method suggested by Farmer et al. (2015). ${ }^{11}$ The solution obtained using the

\footnotetext{
${ }^{10}$ Details on the derivation of the solutions are provided in the Appendix C.

${ }^{11}$ The derivation of the solutions obtained using the method by Farmer et al. (2015) and the proposed augmented representation are provided in Appendix D.
} 


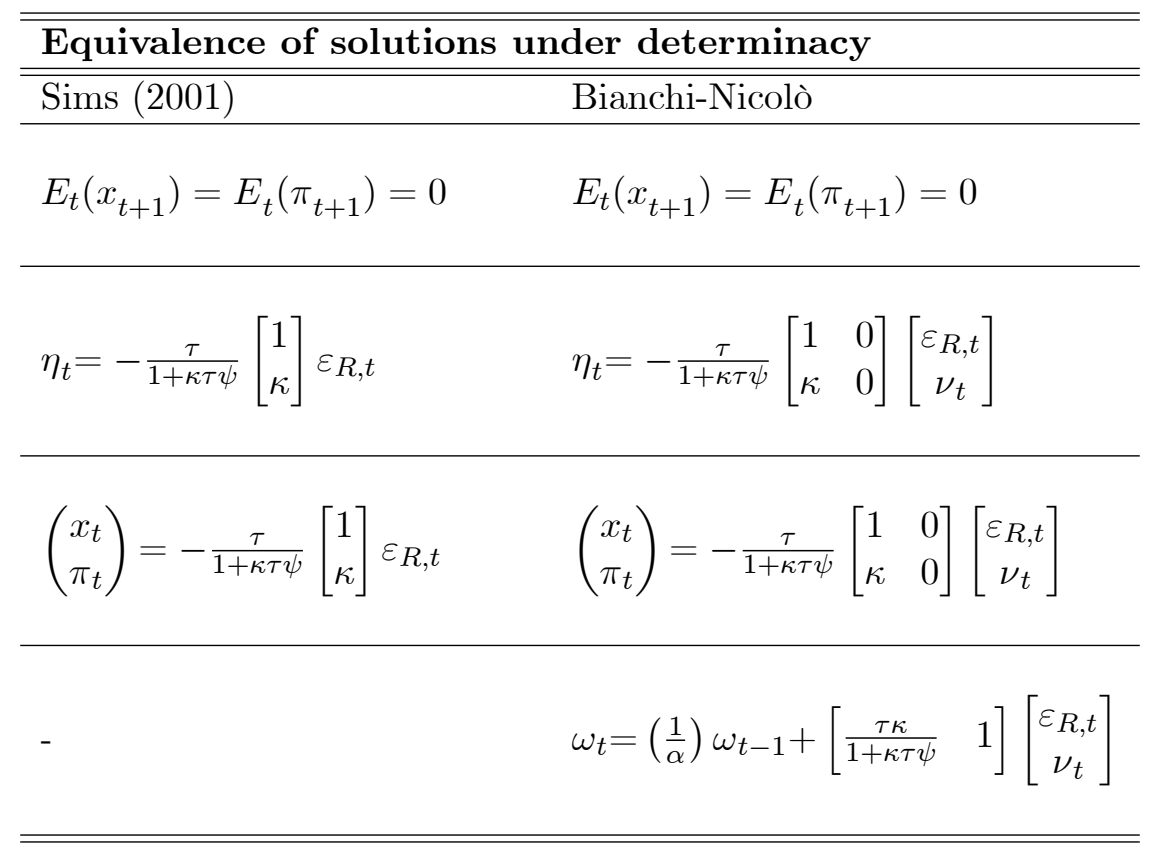

Table 2: Equivalence of solutions under determinacy. The table compares the solution obtained by using our methodology with Sims (2001).

method of Farmer et al. (2015) is equivalent to Lubik and Schorfheide (2003,2004). We use Farmer et al. (2015) solution because easier to compare with our solution. Hence, the solutions that we compare in this section derive from the following two representations:

c) The matrix representation of the LRE model using the methodology of Farmer et al. (2015) when the forecast error for the deviations of inflation from its steady state, $\eta_{2, t}$, is included as newly defined fundamental shock. Given the partition of the matrix $\Pi$ in (58) as $\Pi=$ $\left[\begin{array}{ll}\Pi_{n} & \Pi_{f}\end{array}\right]$, then

$$
\Gamma_{0} X_{t}=\Gamma_{1} X_{t-1}+\Psi_{f} \tilde{\varepsilon}_{t}+\Pi_{n} \eta_{1, t}
$$

where $\tilde{\varepsilon}_{t} \equiv\left(\varepsilon_{t}, \eta_{2, t}\right)^{T}$ and $\Psi_{f} \equiv\left[\begin{array}{ll}\Psi & \Pi_{f}\end{array}\right]$.

d) The proposed augmented representation, equivalent to the representation b) in Section 4.1

$$
\hat{\Gamma}_{0} \hat{X}_{t}=\hat{\Gamma}_{1} \hat{X}_{t-1}+\hat{\Psi} \hat{\varepsilon}_{t}+\hat{\Pi} \eta_{t}
$$


The equilibrium conditions obtained using representations c) and d) are reported in Table 3, where

$$
\underset{4 \times 1}{G} \equiv\left(\begin{array}{c}
-\frac{a_{2}}{2 \kappa} \\
1 \\
-\frac{a_{1} a_{2}}{4 \beta \kappa} \\
\frac{a_{1}}{2 \beta}
\end{array}\right) \quad \underset{4 \times 2}{H} \equiv\left(\begin{array}{cc}
-\frac{2 \beta \tau}{a_{3}} & \frac{2 \kappa \tau(1-\beta \psi)-a_{2}}{a_{3} \kappa} \\
0 & 1 \\
-\frac{\tau a_{2}}{a_{3}} & -\frac{a_{2}(1+\kappa \tau \psi)}{a_{3} \kappa} \\
\frac{2 \kappa \tau}{a_{3}} & -\frac{2(1+\kappa \tau \psi)}{a_{3}}
\end{array}\right)
$$

and $a_{1}=(\beta-\phi+\kappa \tau+1), a_{2}=\left(a_{1}-2\right), a_{3}=\left(a_{1}+2 \phi\right)$ and $\phi=\left[(1+\beta+\kappa \tau)^{2}-4 \beta(1+\kappa \tau \psi)\right]^{-1 / 2}$. To understand the equivalence result, it is useful to compare the linear restrictions imposed on the vector of forecast errors using the augmented representation. In particular, note that our methodology imposes the restriction $\eta_{2, t}=\nu_{t}$. Thus, the solution to the augmented representation sets restrictions on the forecast error, $\eta_{2, t}$, (which has been redefined as fundamental using the methodology of Farmer et al. (2015)) such that it corresponds to the sunspot shock, $\nu_{t}$. Also, to guarantee a bounded solution, restrictions are imposed such that the autoregressive process $\omega_{t}$ equals zero at any time $t$. Therefore, the solutions for the two alternative representations are equivalent.

Also, two relevant comments can be made. First, under indeterminacy the endogenous variables are also affected by the sunspot shock. Second, comparing the form of the matrices under determinacy in Table 2 with those under indeterminacy in Table 3, it is evident that the propagation mechanism differs according to which region of the parameter space is considered.

\section{Applications}

While the previous section provides an analytic example clarifying the equivalence results shown in Section 3, this section highlights the importance of our results for the estimation of LRE models. We consider the three-equation NK model of Lubik and Schorfheide (2004) and we conduct the following exercises. Section 5.1 and Section 5.2 deal with simulated data. In particular, we run two simulations of the model for parameter values which lie in the region of the parameter space associated with determinacy and indeterminacy. Given the two simulations, Section 5.1 assumes that the region of determinacy is known. In Section 5.2, we then assume that the region of determinacy is unknown. In both cases the MCMC algorithm converges to the correct area of the parameter space. Section 5.3 then provides an example on how to implement our methodology when using real data. We consider the data of Lubik and Schorfheide (2004) for the period prior to the appointment of Chairman Paul Volcker, and we retain the assumption that the researcher does not know the region of determinacy. We show that our method enables to successfully recover the same posterior distributions reported by Lubik and Schorfheide (2004), regardless of the region of the parameter space in which the estimation is initialized. Finally, we 


\begin{tabular}{lc}
\hline \hline \multicolumn{1}{l}{ Equivalence of solutions under indeterminacy } \\
\hline \hline Farmer et al. (2015) & Bianchi-Nicolò \\
\hline$E_{t}\left(x_{t+1}\right)=-\frac{a_{2}}{2 \kappa} E_{t}\left(\pi_{t+1}\right)$ & $E_{t}\left(x_{t+1}\right)=-\frac{a_{2}}{2 \kappa} E_{t}\left(\pi_{t+1}\right)$ \\
\hline$\eta_{1, t}=\left[\begin{array}{lll}-\frac{2 \beta \tau}{a_{3}} & \frac{2 \kappa \tau(1-\beta \psi)-a_{2}}{a_{3} \kappa}\end{array}\right]\left[\begin{array}{c}\varepsilon_{R, t} \\
\eta_{2, t}\end{array}\right]$ & $\eta_{t}=\left[\begin{array}{cc}-\frac{2 \beta \tau}{a_{3}} & \frac{2 \kappa \tau(1-\beta \psi)-a_{2}}{a_{3} \kappa} \\
0 & 1\end{array}\right]\left[\begin{array}{c}\varepsilon_{R, t} \\
\nu_{t}\end{array}\right]$ \\
\hline$\left(\begin{array}{c}x_{t} \\
\pi_{t} \\
E_{t}\left(x_{t+1}\right) \\
E_{t}\left(\pi_{t+1}\right)\end{array}\right)={ }_{4 \times 1}^{G} E_{t-1}\left(\pi_{t}\right)+{ }_{4 \times 2}^{H}\left[\begin{array}{c}\varepsilon_{R, t} \\
\eta_{2, t}\end{array}\right]$ & $\left(\begin{array}{c}x_{t} \\
\pi_{t} \\
E_{t}\left(x_{t+1}\right) \\
E_{t}\left(\pi_{t+1}\right)\end{array}\right)={ }_{4 \times 1}^{G} E_{t-1}\left(\pi_{t}\right)+H_{4 \times 2}\left[\begin{array}{c}\varepsilon_{R, t} \\
\nu_{t}\end{array}\right]$ \\
\hline
\end{tabular}

Table 3: Equivalence of solutions under indeterminacy. The table compares the solution obtained by using our methodology with Farmer et al. (2015). This last method, in turn, returns the same solution obtained by applying the methods of Lubik and Schorfheide $(2003,2004)$.

run the estimation several times and verify that the results presented below hold across all of them.

We consider the NK model estimated by Lubik and Schorfheide (2004). The model is described by equations $(66) \sim(71)$ and consists of a dynamic IS curve

$$
x_{t}=E_{t}\left(x_{t+1}\right)-\tau\left(R_{t}-E_{t}\left(\pi_{t+1}\right)\right)+g_{t},
$$

a NK Phillips curve

$$
\pi_{t}=\beta E_{t}\left(\pi_{t+1}\right)+\kappa\left(x_{t}-z_{t}\right),
$$

and a Taylor rule,

$$
R_{t}=\rho_{R} R_{t-1}+\left(1-\rho_{R}\right)\left[\psi_{1} \pi_{t}+\psi_{2}\left(x_{t}-z_{t}\right)\right]+\varepsilon_{R, t} .
$$


The demand shock, $g_{t}$, and the supply shock, $z_{t}$, follow univariate $\operatorname{AR}(1)$ processes

$$
\begin{aligned}
& g_{t}=\rho_{g} g_{t-1}+\varepsilon_{g, t}, \\
& z_{t}=\rho_{z} z_{t-1}+\varepsilon_{z, t},
\end{aligned}
$$

where the standard deviations of the fundamental shocks $\varepsilon_{g, t}, \varepsilon_{z, t}$ and $\varepsilon_{R, t}$ are denoted by $\sigma_{g}, \sigma_{z}$ and $\sigma_{R}$, respectively. As in Lubik and Schorfheide (2004), we allow for the correlation between demand and supply shocks, $\rho_{g z}$, to be nonzero. The rational expectation forecast errors are defined as

$$
\eta_{1, t}=x_{t}-E_{t-1}\left[x_{t}\right], \quad \eta_{2, t}=\pi_{t}-E_{t-1}\left[\pi_{t}\right] .
$$

We define the vector of endogenous variables as $X_{t} \equiv\left(x_{t}, \pi_{t}, R_{t}, E_{t}\left(x_{t+1}\right), E_{t}\left(\pi_{t+1}\right), g_{t}, z_{t}\right)^{T}$, the vectors of fundamental shocks and non-fundamental errors,

$$
\varepsilon_{t}=\left(\varepsilon_{R, t}, \varepsilon_{g, t}, \varepsilon_{z, t}\right)^{T}, \quad \eta_{t}=\left(\eta_{1, t}, \eta_{2, t}\right)^{T}
$$

and the vector of parameters

$$
\theta=\left(\psi_{1}, \psi_{2}, \rho_{R}, \beta, \kappa, \tau, \rho_{g}, \rho_{z}, \sigma_{g}, \sigma_{z}, \sigma_{R}, \rho_{g z}, \rho_{g R}, \rho_{z R}\right)^{T} .
$$

This leads to the following representation of the model,

$$
\Gamma_{0}(\theta) X_{t}=\Gamma_{1}(\theta) X_{t-1}+\Psi(\theta) \varepsilon_{t}+\Pi(\theta) \eta_{t}
$$

The LRE model in (72) is determinate when the following analytic condition is satisfied, $\left|\psi^{*}\right|>1$, where $\psi^{*} \equiv \psi_{1}+\frac{(1-\beta)}{\kappa} \psi_{2}$. However, when the model is indeterminate, $0<\left|\psi^{*}\right| \leq 1$, the system is characterized by one degree of indeterminacy $(m=1)$ since there are two expectational variables $\left\{E_{t}\left(x_{t+1}\right), E_{t}\left(\pi_{t+1}\right)\right\}^{T}$ and only one root outside the unit circle. The methodology we propose consists in augmenting the representation of the model in (72) with the autoregressive process

$$
\omega_{t}=\left(\frac{1}{\alpha}\right) \omega_{t-1}+\nu_{t}-\eta_{2, t}
$$

Hence, we define a new vector of endogenous variables $\hat{X}_{t} \equiv\left(X_{t}, \omega_{t}\right)^{T}$ and a newly defined vector of exogenous shocks as $\hat{\varepsilon}_{t} \equiv\left(\varepsilon_{t}, \nu_{t}\right)^{T}=\left(\varepsilon_{R, t}, \varepsilon_{g, t}, \varepsilon_{z, t}, \nu_{t}\right)^{T}$. The system in (72) and (73) can then be written as

$$
\hat{\Gamma}_{0} \hat{X}_{t}=\hat{\Gamma}_{1} \hat{X}_{t-1}+\hat{\Psi} \hat{\varepsilon}_{t}+\hat{\Pi} \eta_{t}
$$

As in Lubik and Schorfheide (2004), the vector of observables, $\mathbf{y}_{t}=\left\{x_{o b s, t}, \pi_{o b s, t}, R_{o b s, t}\right\}$, consists 
of

1. $x_{o b s, t}$ the percentage deviations of (log) real GDP per capita from an HP-trend;

2. $\pi_{o b s, t}$ the annualized percentage change in the Consumer Price Index for all Urban Consumers;

3. $R_{o b s, t}$ the annualized percentage average Federal Funds Rate.

The measurement equations are described by

$$
\mathbf{y}_{t}=\left[\begin{array}{c}
0 \\
\pi^{*} \\
\pi^{*}+r^{*}
\end{array}\right]+\left[\begin{array}{ccccccc}
1 & 0 & 0 & 0 & 0 & 0 & 0 \\
0 & 4 & 0 & 0 & 0 & 0 & 0 \\
0 & 0 & 4 & 0 & 0 & 0 & 0
\end{array}\right] X_{t}
$$

where $\pi^{*}$ and $r^{*}$ are annualized steady-state inflation and real interest rates expressed in percentages. The discount factor, $\beta$ is a function of the annualized real interest rate in steady-state $r^{*}$ (i.e. $\left.\beta=\left(1+r^{*}\right)^{-1 / 4}\right)$. We then simulate the model under both determinacy and indeterminacy and Table 4 reports the parameter values used for the simulations. ${ }^{12}$

While under determinacy we set $\psi_{1}=2.1$ (thus guaranteeing $\left|\psi^{*}\right|>1$ ), for the simulation under indeterminacy we impose $\psi_{1}=0.7$ for which $0<\left|\psi^{*}\right|<1$. Also, under indeterminacy we use the values for the standard deviation of the sunspot shock and its correlation with the fundamental shocks reported in Farmer et al. (2015). Finally, Table 5 reports the prior distributions used for the estimation exercises in the following sections.

\subsection{Known region of determinacy}

In this section, we assume that the region of determinacy is known. We show that our augmented representation accommodates with a single framework both the case of determinacy and indeterminacy. This feature of our solution method makes it possible for the optimization algorithm to search over the entire parameter space, therefore increasing the probability of finding the posterior mode. As explained in An and Schorfheide (2007), the posterior mode is a crucial object for Bayesian inference. First, the posterior mode is often used as a point estimate for the parameters of the model. Second, it is often used as a starting point for the MetropolisHastings algorithm. Finally, a scaled version of the inverse of the Hessian matrix evaluated at

\footnotetext{
${ }^{12}$ The parameter values are those that we estimate in the following section using the data of Lubik and Schorfheide (2004) for the Pre-Volcker period. These estimates are in line with those that the authors report. Also, for the purpose of this paper changing the values assigned to the parameters which are not directly related to the analytic condition defining the region of determinacy is irrelevant.
} 


\begin{tabular}{ccc}
\hline \hline \multicolumn{3}{c}{ Parameter values for simulations } \\
\hline \hline Parameter & Determinacy & Indeterminacy \\
\hline$\psi_{1}$ & 2.1 & 0.73 \\
$\psi_{2}$ & 0.16 & 0.16 \\
$\rho_{R}$ & 0.67 & 0.67 \\
\hline$\pi^{*}$ & 4.03 & 4.03 \\
$r^{*}$ & 1.22 & 1.22 \\
$\kappa$ & 0.86 & 0.86 \\
$\tau^{-1}$ & 1.61 & 1.61 \\
$\rho_{g}$ & 0.77 & 0.77 \\
$\rho_{z}$ & 0.78 & 0.78 \\
\hline$\sigma_{R}$ & 0.22 & 0.22 \\
$\sigma_{g}$ & 0.24 & 0.24 \\
$\sigma_{z}$ & 1.10 & 1.10 \\
$\rho_{g z}$ & 0.46 & 0.46 \\
\hline$\sigma_{\nu}$ & - & 0.24 \\
$\rho_{R \nu}$ & - & -0.19 \\
$\rho_{g \nu}$ & - & 0.15 \\
$\rho_{z \nu}$ & - & -0.21 \\
\hline \hline
\end{tabular}

Table 4: The table reports the parameter values used for the simulations.

the posterior mode is often used as the covariance matrix for the proposal distribution in the Metropolis-Hastings algorithm.

Since we assume that the region of determinacy $\left|\psi^{*}\right|>1$ is known, we set $\alpha \equiv\left|\psi^{*}\right|$. This assumption implies that when the model is determinate, the autoregressive process is stable and the solution is equivalent to the solution of the original model (72). On the other hand, when the model is indeterminate (i.e. $0<\left|\psi^{*}\right| \leq 1$ ), the autoregressive process is unstable, satisfying the Blanchard-Kahn condition. The assumption $\alpha \equiv\left|\psi^{*}\right|$ enables to search for the posterior mode over the entire parameter space.

First, we consider the simulation of the model under determinacy, and we compute the posterior mode of the model parameters using three different representations of the Lubik and Schorfheide (2004) model. We consider the augmented representation proposed in this paper, the representation of the model under determinacy using Sims' (2001) algorithm, and the representation of the model under indeterminacy using the methodology of Farmer et al. (2015). ${ }^{13}$ Figure 1 reports the posterior mode (vertical line) and how the posterior varies to changes in the parameter $\psi_{1}$, while keeping the other structural parameters at their posterior mode estimates. While panel (a)

\footnotetext{
${ }^{13}$ As in Section 4.2, we apply the methodology of Farmer et al. (2015) by redefining the forecast error for inflation, $\eta_{2, t}$, as fundamental shock, that is $\Gamma_{0} X_{t}=\Gamma_{1} X_{t-1}+\Psi_{f} \tilde{\varepsilon}_{t}+\Pi_{n} \eta_{1, t}$, where $\tilde{\varepsilon}_{t}=\left(\varepsilon_{R, t}, \eta_{2, t}\right)^{\prime}$ and $\Pi=\left[\Pi_{n} \quad \Pi_{f}\right]$.
} 


\begin{tabular}{cccccc}
\hline \hline \multicolumn{6}{c}{ Prior distribution for model parameters } \\
\hline \hline Name & Range & Density & Mean & Std. Dev. & $90 \%$ interval \\
\hline$\psi_{1}$ & $R^{+}$ & Gamma & 1.1 & 0.50 & {$[0.43,2.03]$} \\
$\psi_{2}$ & $R^{+}$ & Gamma & 0.25 & 0.15 & {$[0.06,0.54]$} \\
$\rho_{R}$ & {$[0,1)$} & Beta & 0.50 & 0.20 & {$[0.17,0.83]$} \\
\hline$\pi^{*}$ & $R^{+}$ & Gamma & 4.00 & 2.00 & {$[1.35,7.75]$} \\
$r^{*}$ & $R^{+}$ & Gamma & 2.00 & 1.00 & {$[0.69,3.86]$} \\
$\kappa$ & $R^{+}$ & Gamma & 0.50 & 0.20 & {$[0.22,0.87]$} \\
$\tau^{-1}$ & $R^{+}$ & Gamma & 2.00 & 0.50 & {$[1.25,2.88]$} \\
$\rho_{g}$ & {$[0,1)$} & Beta & 0.70 & 0.10 & {$[0.52,0.85]$} \\
$\rho_{z}$ & {$[0,1)$} & Beta & 0.70 & 0.10 & {$[0.52,0.85]$} \\
\hline$\sigma_{R}$ & $R^{+}$ & Inverse Gamma & 0.31 & 0.16 & {$[0.14,0.60]$} \\
$\sigma_{g}$ & $R^{+}$ & Inverse Gamma & 0.38 & 0.20 & {$[0.17,0.74]$} \\
$\sigma_{z}$ & $R^{+}$ & Inverse Gamma & 1.00 & 0.52 & {$[0.47,1.95]$} \\
\hline$\rho_{g z}$ & {$[-1,1]$} & Uniform & 0.00 & 0.58 & {$[-0.90,0.90]$} \\
\hline$\sigma_{\nu}$ & $R^{+}$ & Uniform & 0.5 & 0.29 & {$[0.05,0.95]$} \\
$\rho_{R \nu}$ & {$[-1,1]$} & Uniform & 0.00 & 0.58 & {$[-0.90,0.90]$} \\
$\rho_{g \nu}$ & {$[-1,1]$} & Uniform & 0.00 & 0.58 & {$[-0.90,0.90]$} \\
$\rho_{z \nu}$ & {$[-1,1]$} & Uniform & 0.00 & 0.58 & {$[-0.90,0.90]$} \\
\hline \hline
\end{tabular}

Table 5: The table reports the distributions used as priors for the model parameters.

considers the augmented representation, panel (b) and (c) report the plots for the representations under model determinacy and indeterminacy, respectively.

The red dots on the horizontal axis in panel b) and c) indicate parameter values for which, given the chosen model representation, the model could not be solved due to a violation of the Blanchard-Kahn condition. While in panel b) the model violates these conditions for values of the parameter $\psi_{1}$ smaller than 1, panel c) shows that the representation of Farmer et al. (2015) does not allow to solve the model for values of $\psi_{1}$ greater than $1 .{ }^{14}$ Figure 1 highlights that the augmented representation guarantees that the optimization algorithm explores the entire domain of the parameter space. Note that as pointed out by Lubik and Schorfheide (2004) the posterior presents a discrete jump between the two parameter regions. This makes it unlikely that once the correct region of the parameter space is reached, the estimation algorithm will leave such region. We will further elaborate on this point in Section 6 .

Similarly, when using the Metropolis-Hastings algorithm, candidate parameter values can be drawn from both the determinacy and the indeterminacy region. However, once the algorithm converges to the "correct" area of the parameter space, it is unlikely to leave it. This is reflected

\footnotetext{
${ }^{14}$ The violation of the Blanchard-Kahn conditions for values of $\psi_{1}$ close to 1 results from the values chosen for the simulation. Indeed, the term $\frac{(1-\beta)}{\kappa} \psi_{2} \approx 0$, thus implying that the region of determinacy is approximated by the following condition $\psi^{*} \approx \psi_{1}>1$.
} 
Posterior function and posterior mode for parameter $\psi_{1}$ (determinacy)
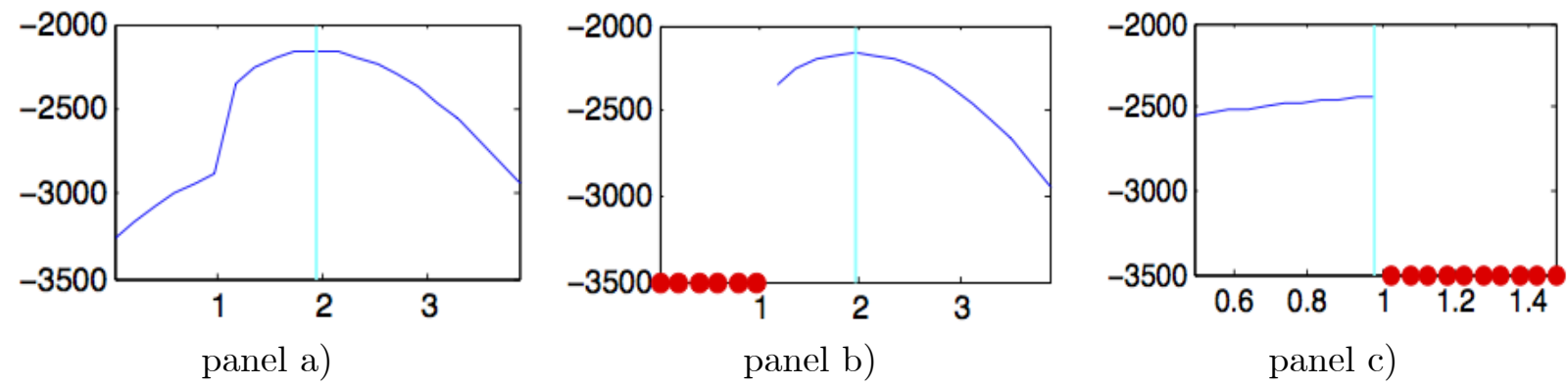

Figure 1: The figure reports the posterior function and posterior mode for the parameter $\psi_{1}$ for the augmented representation (panel (a)), the representation under determinacy (panel (b)) and under indeterminacy (panel (c)).

in Table 6, that reports the mean and the $90 \%$ probability interval of the posterior distributions. ${ }^{15}$ The posterior estimates indicate that the true parameter values are recovered under the augmented representation. All the parameter values used to simulate the model fall within the $90 \%$ probability intervals of the posterior distributions.

We perform the same estimation exercise using the simulation of the model under indeterminacy. Figure 2 plots how the posterior varies with $\psi_{1}$ while the other parameters are constant at their posterior mode estimates. As before, the vertical line reports the corresponding posterior mode. Figure 2 provides similar evidence as in Figure 1. Panel (a), panel (b) and panel (c) refer to the augmented representation and the representation under determinacy and under indeterminacy, respectively.

Contrary to the alternative representations, the proposed augmented representation ensures to run the optimization routine to compute the posterior mode over the entire parameter space. Reasonably, the shape of the maximized functions in panel (a) of Figure 2 mirrors the plot of panel (a) in Figure 1, with the difference that now the peak for the posterior occurs in the indeterminacy region. Table 7 reports the mean and $90 \%$ probability of the posterior distribution. Also in this case, we recover the true parameter values by using the proposed augmented representation. Since the posterior estimates indicate that the model is characterized by indeterminacy, we report the standard error of the sunspot shock, $\sigma_{\nu}$, and its covariance with the fundamental shocks (i.e. $\left.\rho_{\nu R}, \rho_{\nu g}, \rho_{\nu z}\right)$.

\footnotetext{
${ }^{15}$ Since the posterior estimates satisfy the analytic condition for determinacy, the endogenous variables, $X_{t}$, are not a function of the sunspot shock and we therefore do not report the estimates of the standard error of the sunspot shock and its correlation with the fundamental shocks.
} 


\begin{tabular}{lccc}
\hline \hline \multicolumn{3}{c}{ Posterior estimates, simulation } & under determinacy \\
\hline \hline & True values & \multicolumn{2}{c}{ Posterior estimates } \\
\hline & & Mean & $90 \%$ probability interval \\
\hline$\psi_{1}$ & 2.1 & 1.90 & {$[1.59,2.22]$} \\
$\psi_{2}$ & 0.16 & 0.34 & {$[0.03,0.62]$} \\
$\rho_{R}$ & 0.67 & 0.67 & {$[0.64,0.70]$} \\
\hline$\pi^{*}$ & 4.03 & 4.17 & {$[3.95,4.40]$} \\
$r^{*}$ & 1.22 & 1.39 & {$[1.13,1.65]$} \\
$\kappa$ & 0.86 & 0.71 & {$[0.44,0.98]$} \\
$\tau^{-1}$ & 1.61 & 1.66 & {$[1.22,2.10]$} \\
$\rho_{g}$ & 0.77 & 0.75 & {$[0.70,0.79]$} \\
$\rho_{z}$ & 0.78 & 0.77 & {$[0.73,0.82]$} \\
\hline$\sigma_{R}$ & 0.22 & 0.21 & {$[0.20,0.22]$} \\
$\sigma_{g}$ & 0.24 & 0.26 & {$[0.22,0.30]$} \\
$\sigma_{z}$ & 1.10 & 1.07 & {$[0.98,1.15]$} \\
$\rho_{g z}$ & 0.46 & 0.35 & {$[0.15,0.58]$} \\
\hline \hline
\end{tabular}

Table 6: The table reports the posterior distributions obtained by estimating the model using the simulation under determinacy.

\subsection{Unknown region of determinacy}

In this section, we assume that the region of determinacy, $\left|\psi^{*}\right|>1$, is unknown. By considering this case, we show that our methodology can be used to study LRE models for which it is nontrivial to derive an analytic condition describing the region of determinacy. Thus, the approach allows a researcher to estimate medium- and large-scale LRE models that could potentially be characterized by indeterminacy. Our methodology allows the researcher to conduct Bayesian inference on the model parameters over the entire parameter space and to compute their posterior estimates which could potentially lie in both regions of determinacy and indeterminacy.

The assumption that the region of determinacy is unknown implies that it is no longer possible to impose $\alpha \equiv\left|\psi^{*}\right|$. To ensure that the Metropolis-Hastings algorithm explores the entire parameter space, we assume a uniform distribution over the interval $[0,2]$ as a prior distribution for the parameter $\alpha .{ }^{16}$ Equivalently, we assume that there is an equal probability of making draws of $\alpha$ from the interval $[0,1)$ as well as from the interval [1,2]. Draws of $\alpha$ from [1,2] combined with draws of the other parameters $\theta$ which satisfy the condition $\left|\psi^{*}\right|>1$ ensure to solve the augmented representation under determinacy. Similarly, draws of $\alpha$ from $[0,1)$ combined with draws of the other parameters of interest $\theta$ such that $0<\left|\psi^{*}\right| \leq 1$ ensure to solve the proposed

\footnotetext{
${ }^{16}$ The choice of the interval $[0,2]$ is arbitrary. For any value $0<a \leq 1$, it is sufficient to specify an interval $[-a+1, a+1]$ as the domain of the uniform distribution.
} 
Posterior function and posterior mode for parameter $\psi_{1}$ (indeterminacy)
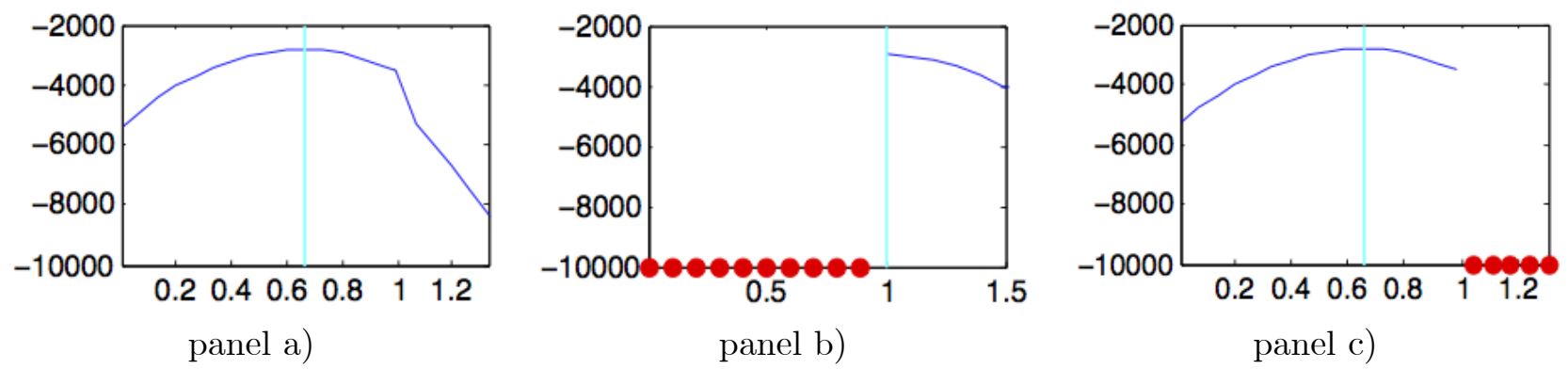

Figure 2: The figure reports the posterior function and posterior mode for the parameter $\psi_{1}$ for the augmented representation (panel (a)), the representation under determinacy (panel (b)) and under indeterminacy (panel (c)).

representation under indeterminacy. ${ }^{17}$

Importantly, the same intuition described in Section 5.1 still holds. The Metropolis-Hastings algorithm makes draws of $\alpha$ and $\theta$ which could solve the augmented representation under determinacy and indeterminacy, and it compares the posterior obtained for draws in both regions. Having specified the prior for $\alpha$, we estimate the augmented representation using the same two simulations of the data as in Section 5.1. We first estimate the augmented representation of the model using the data simulated under determinacy and the same prior distributions reported in Table 5.

The posterior distribution for the parameter $\alpha$ is plotted in Figure 3. Two remarks should be made. First, the posterior distribution is distributed over the interval [1,2], thus providing evidence that the Metropolis-Hastings algorithm explores the entire parameter space and successfully recovers the information contained in the simulated data about model determinacy. Second, the posterior distribution approximates a uniform distribution over the same interval. This result is in line with the non-identifiability of the parameter $\alpha$ stated in Corollary 3. Finally, the posterior mean and $90 \%$ probability intervals of the parameters are the same as those reported in Table 6 when we assume that the region of determinacy is known. As in Section 5.1, the estimation procedure conducted on our augmented representation recovers the true parameter values. Also in this case, the results are independent of the initial parametrization used to start the algorithm. Regardless of whether the starting parametrization is in the 'correct' region, the estimation al-

\footnotetext{
${ }^{17}$ The virtue of using a continuous distribution for $\alpha$ and treating it as any other parameter of the model is that the algorithm can be easily implemented in Dynare. However, the efficiency of the algorithm could be improved by using a discrete distribution for $\alpha$ given that the only thing that matters is if this parameter is inside or outside the unit circle. Furthermore, the MCMC algorithm could be modified to allow for the possibility that whenever the augmented model does not have a solution, the value of $\alpha$ is flipped.
} 


\begin{tabular}{lccc}
\hline \hline \multicolumn{3}{c}{ Posterior estimates, simulation } & under indeterminacy \\
\hline \hline & True values & \multicolumn{2}{c}{ Posterior estimates } \\
\hline$\psi_{1}$ & & Mean & $90 \%$ probability interval \\
$\psi_{2}$ & 0.73 & 0.76 & {$[0.71,0.81]$} \\
$\rho_{R}$ & 0.16 & 0.14 & {$[0.02,0.26]$} \\
\hline$\pi^{*}$ & 0.67 & 0.69 & {$[0.65,0.75]$} \\
$r^{*}$ & 1.03 & 3.28 & {$[2.02,4.56]$} \\
$\kappa$ & 1.22 & 1.42 & {$[1.13,1.72]$} \\
$\tau^{-1}$ & 0.86 & 0.77 & {$[0.49,1.04]$} \\
$\rho_{g}$ & 1.61 & 1.89 & {$[1.32,2.50]$} \\
$\rho_{z}$ & 0.77 & 0.76 & {$[0.70,0.82]$} \\
\hline$\sigma_{R}$ & 0.78 & 0.77 & {$[0.72,0.81]$} \\
$\sigma_{g}$ & 0.22 & 0.21 & {$[0.20,0.22]$} \\
$\sigma_{z}$ & 0.24 & 0.23 & {$[0.17,0.29]$} \\
$\rho_{g z}$ & 1.10 & 1.06 & {$[0.98,1.14]$} \\
\hline$\sigma_{\nu}$ & 0.46 & 0.49 & {$[0.20,0.79]$} \\
$\rho_{R \nu}$ & 0.24 & 0.25 & {$[0.17,0.32]$} \\
$\rho_{g \nu}$ & -0.19 & -0.22 & {$[-0.37,-0.07]$} \\
$\rho_{z \nu}$ & 0.15 & 0.22 & {$[-0.25,0.72]$} \\
\hline \hline
\end{tabular}

Table 7: The table reports the posterior distributions obtained by estimating the model using the simulation under indeterminacy.

gorithm successfully recovers the true parameter values used for the simulations. However, the speed of the convergence for the parameter estimates might be affected.

The estimation of the augmented representation using simulated data under indeterminacy delivers a mirrored posterior distribution for the parameter $\alpha$ (Figure 4). In this case, the posterior distribution of the parameter $\alpha$ is distributed over the interval $[0,1)$ and it closely resembles a uniform distribution over the same interval due to its non-identifiability. As for the simulation under determinacy, we obtain the same posterior mean and the $90 \%$ probability interval as for the case of a known region of determinacy reported in Table 7 . Hence, also when we assume that the region is unknown to the researcher, we recover the true parameter values by estimating the augmented representation.

\subsection{Indeterminacy in the 1970s}

This section provides an example on how to implement our methodology when using real data. We retain the assumption that the researcher does not know the region of determinacy, and we show that our method enables the algorithm to jump across the regions of determinacy and 


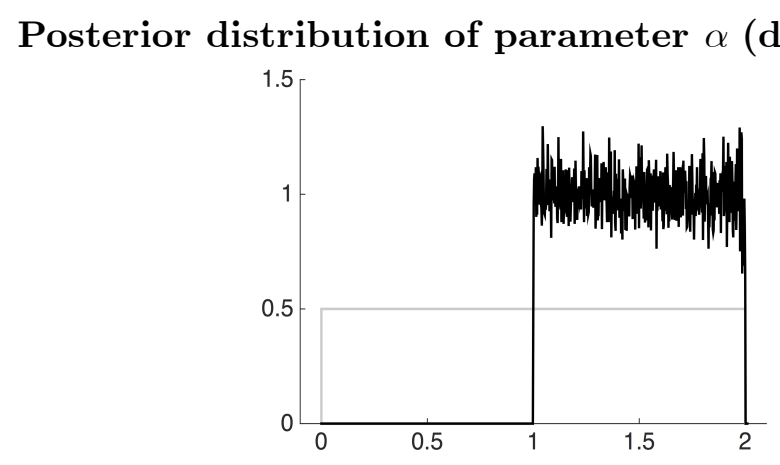

Figure 3: The grey line represents the prior distribution for the parameter $\alpha$. The black line is the posterior distribution.

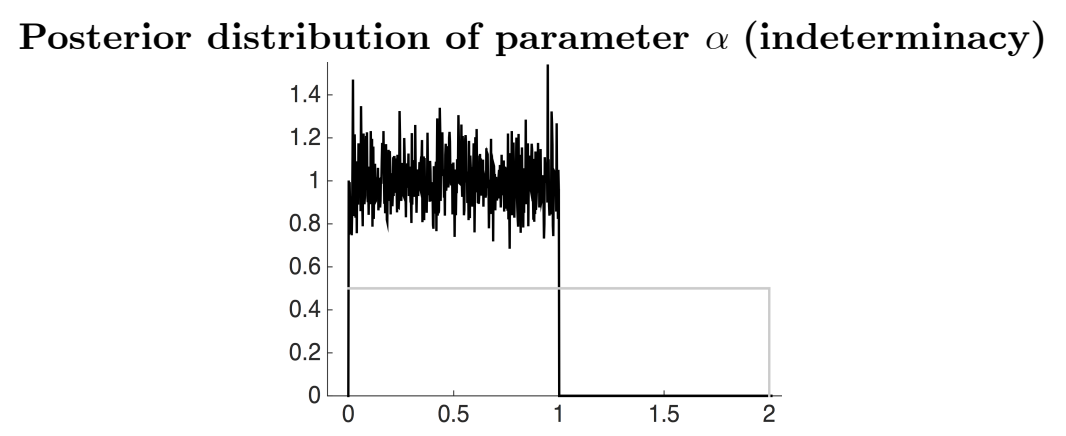

Figure 4: The grey line represents the prior distribution for the parameter $\alpha$. The black line is the posterior distribution. 


\section{Posterior distribution of parameter $\psi_{1}$ and $\alpha$}
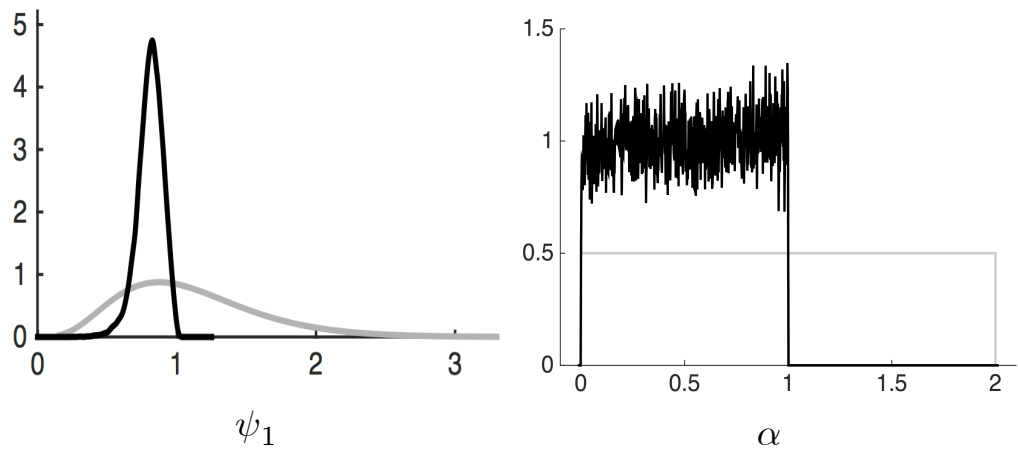

Figure 5: The grey line represents the prior distribution and the black line is the posterior distribution.

indeterminacy, thus facilitating the search for the global maximum in the marginal data density.

We consider both the model and the data that Lubik and Schorfheide (2004) use to test for indeterminacy in U.S. monetary policy. The model is described by equations $(66) \sim(71)$ at the beginning of Section 5 and, as previously explained, we append the process in (73) to obtain the augmented representation that we propose. Finally, equation (75) presents the measurement equations that link the endogenous variables of the model to the data. In the following, we focus on the data for the pre-Volcker period (1960Q1 - 1979Q2) since Lubik and Schorfheide (2004) show that during this period the monetary authority did not respond aggressively enough to changes in inflation, thus not suppressing self-fulfilling inflation expectations.

We proceed by starting the algorithm from initial conditions in both regions of the parameter space and by allowing for a large number of draws. ${ }^{18}$ We verified that this approach guarantees the proper convergence of the posterior distributions for any initial parametrization by repeating this estimation exercise 100 times and successfully recovering the same posterior estimates in each case. Figure 5 reports the posterior distribution for both $\psi_{1}$ and $\alpha$ which clearly favor the indeterminate model regardless of the initial values for the parameters. ${ }^{19}$ Table 8 reports the corresponding posterior mean and $90 \%$ probability interval of the model parameters. As expected, the estimates obtained using our procedure are in line with the empirical results in Lubik and Schorfheide (2004) that we report in the first column. ${ }^{20}$

\footnotetext{
${ }^{18}$ In particular, we run two chains of $1,000,000$ each and discard the first half of the draws.

${ }^{19}$ The prior that we used for the parameter $\psi_{1}$ is the same as in Table 5 and is defined on both regions of determinacy and indeterminacy. This is important for the possibility of the posterior estimates to lie in either region since having a prior distribution which assigns zero probability to either one would also imply that the posterior would have no mass in the same region.

${ }^{20}$ The minor difference in the point estimate of the posterior mean for the correlation between demand and
} 


\begin{tabular}{|c|c|c|c|}
\hline \multicolumn{4}{|c|}{ Posterior estimates } \\
\hline & LSS estimates & Posterio & estimates \\
\hline & & Mean & $90 \%$ probability interval \\
\hline$\alpha$ & - & 0.95 & {$[0.90,0.99]$} \\
\hline$\psi_{1}$ & 0.77 & 0.73 & {$[0.56,0.90]$} \\
\hline$\psi_{2}$ & 0.17 & 0.16 & {$[0.01,0.30]$} \\
\hline$\rho_{R}$ & 0.60 & 0.67 & {$[0.48,0.86]$} \\
\hline$\pi^{*}$ & 4.28 & 4.03 & {$[1.87,6.06]$} \\
\hline$r^{*}$ & 1.13 & 1.22 & {$[0.64,1.78]$} \\
\hline$\kappa$ & 0.77 & 0.86 & {$[0.45,1.26]$} \\
\hline$\tau^{-1}$ & 1.45 & 1.61 & {$[0.93,2.27]$} \\
\hline$\rho_{g}$ & 0.68 & 0.77 & {$[0.66,0.88]$} \\
\hline$\rho_{z}$ & 0.82 & 0.78 & {$[0.68,0.88]$} \\
\hline$\sigma_{R}$ & 0.23 & 0.22 & {$[0.19,0.25]$} \\
\hline$\sigma_{g}$ & 0.27 & 0.25 & {$[0.16,0.32]$} \\
\hline$\sigma_{z}$ & 1.13 & 1.10 & {$[0.93,1.27]$} \\
\hline$\rho_{g z}$ & 0.14 & 0.47 & {$[-0.04,0.95]$} \\
\hline$\sigma_{\nu}$ & - & 0.24 & {$[0.16,0.33]$} \\
\hline$\rho_{R \nu}$ & - & -0.19 & {$[-0.65,0.27]$} \\
\hline$\rho_{g \nu}$ & - & 0.15 & {$[-0.40,0.71]$} \\
\hline$\rho_{z \nu}$ & - & -0.21 & {$[-0.55,0.14]$} \\
\hline
\end{tabular}

Table 8: The table reports the posterior distributions obtained by estimating the model using the data from Lubik and Schorfheide (2004). The terms "-" indicate that the estimates are not directly comparable.

\section{Tips for implementation}

In this section, we present some suggestions for the practical implementation of our method.

Convergence. We repeat the estimation of the model of Lubik and Schorfheide (2004) by using parameters in the "wrong" region of the parameter space and considering only a few $(200,000)$ draws to show the importance of checking convergence before interpreting the estimation results. Figure 6 reports the posterior distribution for the parameter $\psi_{1}$ and $\alpha$ obtained for an initial parametrization close to the Taylor Principle (i.e. we set $\psi_{1}=1.1$ ). At first glance, the posterior distribution of the parameter $\psi_{1}$ would appear to be bimodal. This is consistent with the fact that the proposed augmented representation allows the Metropolis-Hastings algorithm to visit both

supply shocks, $\rho_{g z}$, derives from the prior distribution that we assume for this parameter. While Lubik and Schorfheide (2004) assume a normal prior centered at 0 and with standard deviation 0.4 , we assume a flat, uniform distribution over the interval $(-1,1)$. Nevertheless, both in Lubik and Schorfheide (2004) and in this paper, the estimate of $\rho_{g z}$ is not statistically significant. 
regions of the parameter space. At the same time, the posterior distribution for the parameter $\alpha$ is very similar to the prior distribution, which is specified as a uniform distribution over the interval $[0,2]$. Such a result is just the other side of coin of the posterior for $\psi_{1}$ since the algorithm explores both regions by considering draws of $\alpha$ which are within as well as outside the unit circle.

\section{Posterior distribution of parameter $\psi_{1}$ and $\alpha$}
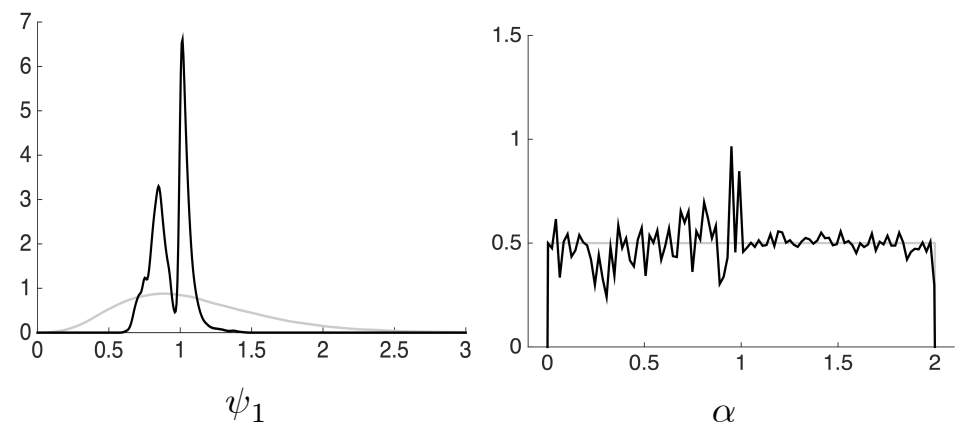

Figure 6: Initial parametrization $\psi_{1}=1.1$. The grey line represents the prior distribution and the black line is the posterior distribution.

A researcher should then verify the occurrence of either of the following two circumstances. This bimodal distribution could arise because the log-likelihood is highly discontinuous between the two regions. In this case, the algorithm could have jumped towards the region where the peak of the posterior lies, without having spent a significant time there. In other words, convergence has not occurred yet. Alternatively, if the log-likelihood function varies smoothly between the two regions of the parameter space, the posterior distribution plotted in Figure 6 could be the result of the algorithm travelling across the two regions multiple times.

We therefore recommend the researcher to analyze the draws of the parameter $\alpha$ which have been accepted during the MCMC algorithm. By inspecting the behavior of the auxiliary parameter $\alpha$, a researcher can detect if the algorithm reached convergence or not. We report the draws that we obtained during our exercise in Figure 7. After approximately 40,000 draws of $\alpha$ in the region of determinacy (i.e. outside the unit circle), the algorithm jumps to the indeterminate region and never visits the determinacy region again.

Figure 6 and 7 suggest that we are in the first case, for which the log-likelihood function is highly discontinuous at the boundary between the two regions. Therefore, the researcher should repeat the estimation exercise, increase the number of draws, and make sure that the parameter $\alpha$ stabilizes on one region of the parameter space. Under different circumstances, the researcher could face the second scenario, for which the log-likelihood function transitions smoothly between the two regions. In this case, the parameter $\alpha$ would repeatedly transition between the two areas 


\section{Draws of the parameter $\alpha$}

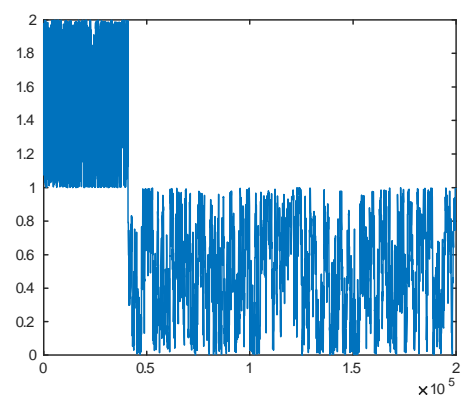

Figure 7: Sequence of draws for $\alpha$ given an initial parametrization $\psi_{1}=1.1$.

of the parameter space and could be used to infer the probability attached to determinacy.

Only (in)determinacy. In some cases, a researcher might want to estimate the model exclusively under determinacy or exclusively under indeterminacy. Our approach easily accommodates this need. If the researcher is only interested in the solution under determinacy, the parameter vector of alpha should be chosen in a way to guarantee stationarity of the auxiliary process (for example, fixing all values of the alphas to 2). Furthermore, all parameters that are relevant only under indeterminacy could be fixed to zero or any other constant, given that they do not affect the fit of the model under determinacy. If instead the researcher is only interested in estimating the model under indeterminacy, the parameters of the auxiliary process can be chosen in a way to guarantee that the correct number of explosive roots are provided. In this case, the parameters describing the properties of the sunspot disturbances should also be estimated.

Model comparison. A researcher might also be interested in comparing the fit of the model under determinacy and under indeterminacy. Model comparison can be conducted by using standard techniques, such as the harmonic mean estimator proposed by Geweke (1999). If the researcher is interested in comparing the same model under determinacy and under indeterminacy, we recommend the following procedure that adapts the approach used by Lubik and Schorfheide (2004):

1. Estimate the model under determinacy by fixing the parameter(s) alpha to a value larger than one in a way that the model is solved only under determinacy. Note that in this case all parameters that pertain to the solution under indeterminacy, such as the volatility of the sunspot shocks, should be restricted to zero (or any other constant). This restriction avoids penalizing the model for extra parameters that do not affect its fit under determinacy.

2. Estimate the model under indeterminacy by fixing the parameter(s) alpha to a value smaller than one in a way that the model is solved only under indeterminacy. Note that in this 
case all parameters that pertain to the solution under indeterminacy, such as the volatility of the sunspot shocks, should be estimated.

3. Use standard methods to compare the fit of the model under determinacy with the fit of the same model under indeterminacy.

\section{Conclusions}

In this paper, we propose a generalized approach to solve and estimate LRE models over the entire parameter space. Our approach accommodates both cases of determinacy and indeterminacy and it does not require the researcher to know the analytic condition describing the region of determinacy or the degrees of indeterminacy.

When a LRE model is characterized by $m$ degrees of indeterminacy, our approach augments it by appending $m$ autoregressive processes whose innovations are linear combinations of a subset of endogenous shocks and a vector of newly defined sunspot shocks. The resulting augmented representation embeds both the solution which is obtained under determinacy using standard solution methods and that delivered by solving the model under indeterminacy using the approach of Lubik and Schorfheide (2003) and equivalently Farmer et al. (2015). We provide an analytical example for the theoretical result using a canonical NK model.

We finally apply our methodology to the NK model in Lubik and Schorfheide (2004). We simulate two series of data under the assumption of model determinacy and indeterminacy and we then estimate our augmented representation for both cases in which the region of determinacy is known or unknown to the researcher. In both case, the parameters used to generate the data are correctly recovered independently of the initial parametrization. This shows that our method is suitable for the estimation of medium- and large-size DSGE model for which the determinacy region is generally unknown. This feature of the solution method is used by Nicolò (2017) to study the possibility of multiple solutions in Smets and Wouters (2007). 


\section{References}

S. An and F. Schorfheide. Bayesian analysis of dsge models. Econometric Reviews, 26(2-4): 113-172, 2007.

J. E. Arias. Determinacy properties of medium-sized New-Keynesian models with trend inflation. 2013. Duke University, mimeo.

J. E. Arias, G. Ascari, N. Branzoli, and E. Castelnuovo. Monetary policy, trend inflation and the Great Moderation: An alternative interpretation - Comment. International Finance Discussion Papers, Board of Governors of the Federal Reserve System, 1127, January 2015.

B. Aruoba and F. Schorfheide. Inflation during and after the zero lower bound, 2015. Prepared for the 2015 Jackson Hole Economic Policy Symposium.

G. Ascari, P. Bonomolo, and H. F. Lopes. Rational sunspots, 2016. Working Paper.

O. J. Blanchard and C. M. Kahn. The solution of linear difference models under rational expectations. Econometrica, 48:1305-1313, 1980.

D. Cass and K. Shell. Do sunspots matter? Journal of Political Economy, 91:193-227, 1983.

L. Christiano, M. Eichenbaum, and C. Evans. Nominal rigidities and the dynamics effects of a shock to monetary policy. Journal of Political Economy, 113:1-45, 2005.

R. Clarida, J. Galí, and M. Gertler. Monetary policy rules and macroeconomic stability: Evidence and some theory. Quarterly Journal of Economics, CXV:147-180, 2000.

O. Coibon and Y. Gorodnichenko. Monetary policy, trend inflation, and the Great Moderation: An Alternative Interpretation. American Economic Review, 101(1):341-370, 2011.

R. E. Farmer, V. Khramov, and G. Nicolò. Solving and estimating indeterminate dsge models. Journal of Economic Dynamics and Control, 54:17-36, 2015.

R. E. A. Farmer and J. T. Guo. Real business cycles and the animal spirits hypothesis. Journal of Economic Theory, 63:42-73, 1994.

R. E. A. Farmer and J.-T. Guo. The econometrics of indeterminacy. Carnegie Rochester Series on Public Policy, 43:225-273, 1995.

J. Geweke. Using simulation methods for bayesian econometric models: Inference, development, and communication. Econometric Reviews, 18(1):1-73, 1999. 
E. P. Herbst and F. Schorfheide. Bayesian Estimation of DSGE Models. Princeton University Press, 2015.

K. L. Judd. Numerical Methods in Economics. MIT Press, Cambridge, Massachusetts, October 1998.

W. R. Kerr and R. G. King. Limits on interest rate rules in is-lm models. Federal Reserve Bank of Richmond Economic Quarterly, 82(2):47-75, 1996.

R. G. King and M. Watson. The solution of singular linear difference systems under rational expectations. International Economic Review, 39(4):1015-1026, 1998.

P. Klein. Using the generalized schur form to solve a mutivariate linear rational expectations model. Journal of Economic Dynamics and Control, 24(10):1405-1423, 2000.

T. A. Lubik and F. Schorfheide. Computing sunspot equilibria in linear rational expectations models. Journal of Economic Dynamics and Control, 28(2):273-285, 2003.

T. A. Lubik and F. Schorfheide. Testing for indeterminacy: An application to u.s. monetary policy. American Economic Review, 94:190-219, 2004.

G. Nicolò. The conduct of U.S. monetary policy in the postwar period, 2017. Working Paper.

S. Schmitt-Grohé and M. Uribe. Solving dynamic general equilibrium models using a secondorder approximation to the policy function. Journal of Economic Dynamics and Control, 28 (4):755-775, 2004.

C. A. Sims. Solving linear rational expectations models. Journal of Computational Economics, 20(1-2):1-20, 2001.

F. Smets and R. Wouters. Shocks and frictions in u.s. business cycles: A bayesian dsge approach. American Economic Review, 97(3):586-606, June 2007. 


\section{Appendix}

\subsection{Appendix A}

In this Appendix, we show how the normalization chosen in Lubik and Schorfheide (2004) maps into the methodology we propose. Recall the following notation: $p$ denotes number of expectational variables, $n$ is the number of explosive roots and $m=(p-n)$ are the corresponding degrees of indeterminacy.

As in Lubik and Schorfheide (2004), consider the following structural model

$$
\Gamma_{0}(\theta) X_{t}=\Gamma_{1}(\theta) X_{t-1}+\Psi(\theta) \varepsilon_{t}+\Pi(\theta) \eta_{t}
$$

where $X_{t}$ is the vector of endogenous variables, $\varepsilon_{t}$ is the vector of exogenous shocks, $\eta_{t}$ is the vector of endogenous shocks and we assume that the matrix $\Gamma_{0}$ is invertible. The system can therefore be written as

$$
X_{t}=\Gamma_{1}^{*}(\theta) X_{t-1}+\Psi^{*}(\theta) \varepsilon_{t}+\Pi^{*}(\theta) \eta_{t}
$$

Lubik and Schorfheide (2004) find that, if it exists, the solution to express the forecast errors as a function of the exogenous shocks $\varepsilon_{t}$ and sunspot shocks $\zeta_{t}$ takes the form

$$
\underset{p \times 1}{\eta_{t}}=\left(\underset{p \times n}{-V_{1} D_{11}^{-1} U_{n \times n}^{T} \tilde{\Psi}_{n \times n n \times \ell}}+\underset{p \times m}{V_{2}} \underset{m \times \ell}{\tilde{M}}\right) \underset{\ell \times 1}{\varepsilon_{t}}+\underset{p \times m}{V_{2}} \underset{m \times m}{M_{\zeta}} \underset{m \times 1}{\zeta_{t}} .
$$

More compactly,

$$
\underset{p \times 1}{\eta_{t}}=\underset{p \times n n+\infty \times \ell \times 1}{V_{1}} \underset{\varepsilon_{t}}{N}+\underset{p \times m}{V_{2}} \underset{m \times \ell}{\tilde{M}} \varepsilon_{\ell \times 1}^{\varepsilon_{t}}+\underset{p \times m}{V_{2}} \underset{m \times m}{M_{\zeta}} \underset{m \times 1}{\zeta_{t}}
$$

where

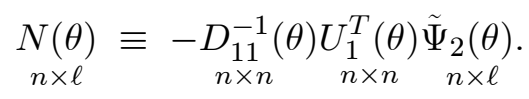

is a function of the parameters of the model.

Combining (77) with (78), the solution that appears in eq. (26) of their paper is

$$
X_{t}=\Gamma_{1}^{*}(\theta) X_{t-1}+\left[\Psi^{*}(\theta)+\Pi^{*}(\theta) V_{1}(\theta) N(\theta)\right] \varepsilon_{t}+\Pi^{*}(\theta) V_{2}(\theta)\left(\tilde{M} \varepsilon_{t}+M_{\zeta} \zeta_{t}\right)
$$

Determinacy Under determinacy, Lubik and Schorfheide (2004) show that $V_{2}(\theta)=0$. Hence, the endogenous variables only respond to exogenous shocks. From (79) the solution is 


$$
X_{t}=\Gamma_{1}^{*}(\theta) X_{t-1}+\left[\Psi^{*}(\theta)+\Pi^{*}(\theta) V_{1}(\theta) N(\theta)\right] \varepsilon_{t}
$$

Using the augmented representation that we propose in this paper, the solution under determinacy is equivalent to (80). Indeed, we are appending a stationary process which constitutes a separate block and does not interact with the endogenous variables of the model.

Indeterminacy Under indeterminacy, Lubik and Schorfheide (2004) show that $V_{2}(\theta) \neq 0$ and the endogenous variables not only respond to exogenous shocks but also to the sunspot shock $\zeta_{t}$. Their solution is in eq. (79) and reported below

$$
X_{t}=\Gamma_{1}^{*}(\theta) X_{t-1}+\left[\Psi^{*}(\theta)+\Pi^{*}(\theta) V_{1}(\theta) N(\theta)\right] \varepsilon_{t}+\Pi^{*}(\theta) V_{2}(\theta)\left(\tilde{M} \varepsilon_{t}+M_{\zeta} \zeta_{t}\right) .
$$

Now we consider the solution under indeterminacy that we obtain using our methodology. Also in our case we assume that $\Gamma_{0}$ is invertible and the system in (76) can be written as

$$
X_{t}=\Gamma_{1}^{*}(\theta) X_{t-1}+\Psi^{*}(\theta) \varepsilon_{t}+\Pi^{*}(\theta) \eta_{t}
$$

Nevertheless, we can show that, if it exists, the solution to express to the endogenous shocks as a function of the exogenous shocks $\varepsilon_{t}$ and sunspot shocks $\nu_{t}$ takes the form

$$
\begin{aligned}
& \underset{p \times 1}{\eta_{t}}=\underset{p \times l}{C_{1}(\theta)} \underset{\ell \times 1}{\varepsilon_{t}}+\underset{p \times m}{C_{2}(\theta) \underset{m \times 1}{\nu_{t}}} .
\end{aligned}
$$

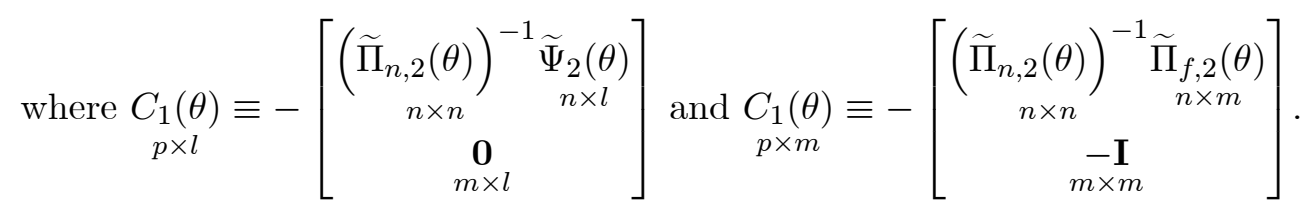

Combining (82) and (83), we obtain the following reduced form

$$
X_{t}=\Gamma_{1}^{*}(\theta) X_{t-1}+\left[\Psi^{*}(\theta)+\Pi^{*}(\theta) C_{1}(\theta)\right] \varepsilon_{t}+\Pi^{*}(\theta) C_{2}(\theta) \nu_{t}
$$

Identification As shown in the previous section, the solution under indeterminacy provided by Lubik and Schorfheide (2004) is derived by combining the following system of equations

$$
X_{t}=\Gamma_{1}^{*}(\theta) X_{t-1}+\Psi^{*}(\theta) \varepsilon_{t}+\Pi^{*}(\theta) \eta_{t}
$$

with the solution for the endogenous shocks as a function of the exogenous shocks $\varepsilon_{t}$ and the 
sunspot shock $\eta_{t}$

$$
\underset{p \times 1}{\eta_{t}}=\underset{p \times n}{V_{1}(\theta)} \underset{n \times \ell}{N(\theta)} \varepsilon_{\ell \times 1}+\underset{p \times m}{V_{2}(\theta) \underset{m \times \ell \times 1}{\tilde{M}}} \varepsilon_{\ell}+\underset{p \times m}{V_{2}(\theta)} \underset{m \times m}{M_{\zeta}} \underset{m \times 1}{\zeta_{t}}
$$

Similarly, the solution obtained using our methodology is derived by combining the same system of equations in (85) with our solution for the endogenous shocks

$$
\underset{p \times 1}{\eta_{t}}=\underset{p \times l}{C_{1}(\theta)} \underset{\ell \times 1}{\varepsilon_{t}}+\underset{p \times m}{C_{2}(\theta)} \underset{m \times 1}{\nu_{t}}
$$

So, in order to understand how the identification strategy implemented in Lubik and Schorfheide (2004) maps into our solution, we only have to study the solutions for the endogenous shocks expressed in (86) and (87).

Lubik and Schorfheide (2004) consider a three equation NK model for which the degree of indeterminacy is at most 1 (i.e. $m=1$ ). This implies that $M_{\zeta}, \zeta_{t}$ and $\nu_{t}$ are scalars. Moreover, the authors assume the following two normalizations

$$
\begin{aligned}
E\left(\varepsilon_{t} \zeta_{t}^{\prime}\right) & =0 \\
M_{\zeta} & =1 .
\end{aligned}
$$

To understand the mapping of these normalizations, we equate the RHS of (86) and (87),

$$
\left(V_{1} N+V_{2} \tilde{M}\right) \varepsilon_{t}+V_{2} M_{\zeta} \zeta_{t}=C_{1} \varepsilon_{t}+C_{2} \nu_{t}
$$

Pre-multiplying by $V_{2}^{\prime}$ and recalling that the matrix $V_{2}$ is orthonormal,

$$
M_{\zeta} \zeta_{t}=\left(V_{2}^{\prime} C_{1}-V_{2}^{\prime} V_{1} N-\tilde{M}\right) \varepsilon_{t}+V_{2}^{\prime} C_{2} \nu_{t}
$$

Post-multiplying by $\zeta_{t}^{\prime}$, we take expectation and consider the normalization in (88) to obtain

$$
M_{\zeta} \sigma_{\zeta}^{2}=\left(V_{2}^{\prime} C_{2}\right) \sigma_{\nu \zeta}
$$

Noting that $\left(\begin{array}{cc}V_{2}^{\prime} & C_{2} \\ 1 \times p & p \times 1\end{array}\right)$ is also a scalar

$$
\sigma_{\nu \zeta}=\frac{M_{\zeta}}{\left(V_{2}^{\prime} C_{2}\right)} \sigma_{\zeta}^{2}
$$


So, the normalization in (88) corresponds to specify a relationship between the covariance of the sunspot shock introduced in Lubik and Schorfheide (2004), $\zeta_{t}$, and the sunspot shock that we specify in our methodology, $\nu_{t}$, with the standard deviation of the sunspot shock, $\zeta_{t}$, scaled by $M_{\zeta}$. Therefore, the normalization $M_{\zeta}=1$ in (89) is such that the relationship in (93) becomes

$$
\sigma_{\nu \zeta}=\left(V_{2}^{\prime} C_{2}\right)^{-1} \sigma_{\zeta}^{2}
$$

\subsection{Appendix B}

We prove the equivalence between the parametrization of the Lubik-Schorfheide indeterminate equilibrium $\theta^{L S} \in \Theta^{L S}$ and the Bianchi-Nicolò equilibrium parametrized by $\theta^{B N} \in \Theta^{B N}$. In particular, we show that there is a unique mapping between the linear restrictions imposed in each of the two methodologies on the forecast errors to guarantee the existence of at least a bounded solution. As shown in Section 3.2.1, the method by Lubik and Schorfheide (2003) imposes the following restrictions on the non-fundamental shocks, $\eta_{t}$, as a function of the exogenous shocks, $\varepsilon_{t}$, and the sunspot shocks introduced in their specification, $\zeta_{t}$,

$$
\underset{p \times 1}{\eta_{t}}=\left(\underset{p \times n n \times \ell}{V_{1}} \underset{p \times m}{N}+\underset{\substack{m \times \ell \\ m \times \ell}}{\widetilde{M}} \underset{\substack{V^{2} \\ \ell \times 1}}{\varepsilon_{t}}+\underset{p \times m}{V_{2}} \underset{m \times 1}{\zeta_{t}} .\right.
$$

Using the methodology proposed in this paper, Section 3.2.2 shows that the restrictions on the non-fundamental shocks, $\eta_{t}$, as a function of the exogenous shocks, $\varepsilon_{t}$, and the sunspot shocks, $v_{t}$, are

$$
\underset{p \times 1}{\eta_{t}}=\underset{p \times \ell \times 1}{C_{1} \varepsilon_{t}}+\underset{p \times m m \times 1}{C_{2}} \underset{p}{\nu_{t}}
$$

where

$$
C_{1} \equiv-\left[\begin{array}{c}
\widetilde{\Pi}_{n, 2}^{-1} \widetilde{\Psi}_{2} \\
\mathbf{0}
\end{array}\right] \quad \text { and } \quad C_{2} \equiv-\left[\begin{array}{c}
\widetilde{\Pi}_{n, 2}^{-1} \widetilde{\Pi}_{f, 2} \\
-\mathbf{I}
\end{array}\right]
$$

Post-multiplying equation (95) and (96) by $\varepsilon_{t}^{T}$ and taking expectation,

$$
\begin{aligned}
& \Omega_{\eta \varepsilon}=\underset{p \times l}{V_{1} \underset{p \times n}{N} N \times \Omega_{\ell \varepsilon \varepsilon}}+\underset{p \times m}{V_{2}} \underset{m \times \ell}{\widetilde{M}} \Omega_{\ell \varepsilon \varepsilon}, \\
& \underset{p \times l}{\Omega_{\eta \varepsilon}}=\underset{p \times \ell \ell \times l}{C_{1} \Omega_{\varepsilon \varepsilon}}+\underset{p \times m m \times l}{C_{2} \Omega_{\nu \varepsilon}}
\end{aligned}
$$


Pre-multiplying by $V_{2}^{T}$ and equating the equations,

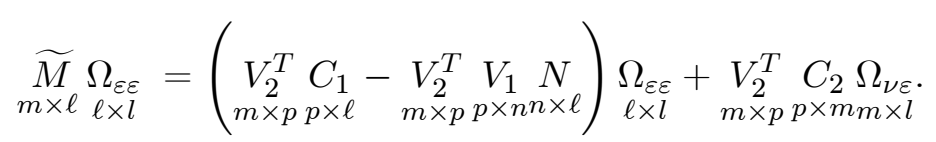

Using the properties of the vec operator, the following result holds

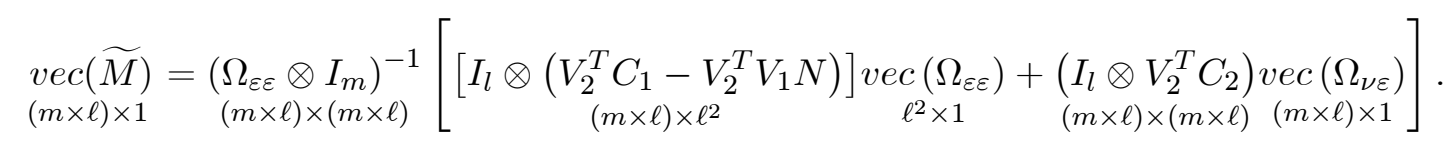

Also, considering again equation (95) and (96), we post-multiply by $\zeta_{t}^{T}$ and take expectation,

$$
\begin{aligned}
& \underset{p \times m}{\Omega_{\eta \zeta}}=\underset{p \times m}{V_{2}} \underset{m \times m}{\Omega_{\zeta \zeta}}, \\
& \underset{p \times m}{\Omega_{\eta \zeta}}=\underset{p \times m_{m \times m}}{C_{2} \Omega_{\nu \zeta}}
\end{aligned}
$$

Pre-multiplying both equations by $V_{2}^{T}$ and equating them,

$$
\underset{m \times m}{\Omega_{\zeta \zeta}}=\underset{m \times m}{\Omega_{\zeta \nu}} \underset{m \times m}{\left(V_{2}^{T} C_{2}\right)^{T}}
$$

Finally, to obtain an expression for $\Omega_{\zeta \nu}$, we post-multiply equation (95) and (96) by $\nu_{t}^{T}$ and taking expectations

$$
\begin{aligned}
& \Omega_{p \times m}=\left(\underset{p \times n n n \times \ell}{V_{1}} \underset{p \times m}{N}+\underset{m \times \ell}{V_{2}} \underset{m}{\widetilde{M}}\right) \Omega_{\ell \times m}+\underset{p \times m_{m \times m}}{V_{2 \times m}} \Omega_{\zeta \nu}, \\
& \underset{p \times m}{\Omega_{\eta \nu}}=\underset{p \times \ell \times \times m}{C_{1} \Omega_{\varepsilon \nu}}+\underset{p \times m m \times m}{C_{2} \Omega_{\nu \nu}}
\end{aligned}
$$

Pre-multiplying both equations by $V_{2}^{T}$ and solving for $\Omega_{\zeta \nu}$,

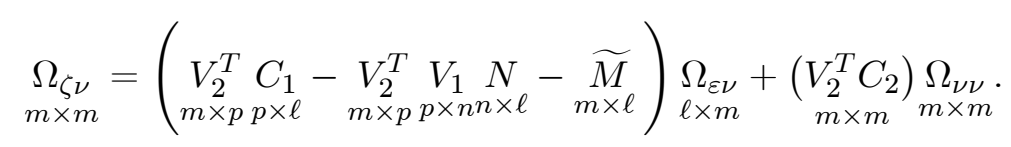

Post-multiplying (99) by $\underset{m \times m}{\left(V_{2}^{T} C_{2}\right)^{T}}$ and using (98), then

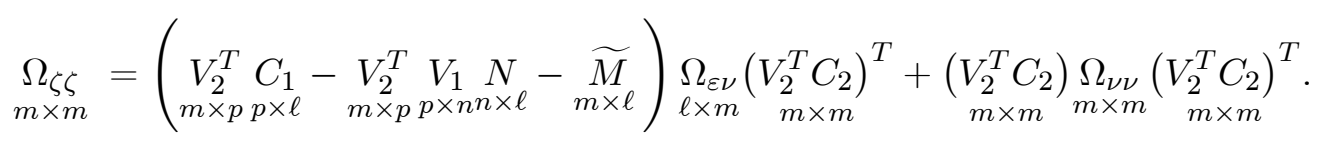

Therefore, equation (97) and (100) define the one-to-one mapping between the parametrization 
in Lubik and Schorfheide $\left\{\Theta, \Theta^{L S}\right\}$ and the parametrization in Bianchi and Nicolò $\left\{\Theta, \Theta^{B N}\right\}$.

\subsection{Appendix $\mathrm{C}$}

In this section, we provide the derivation for the solutions under the two alternative representations discussed in Section 4.1 are provided.

a) Under determinacy, it is possible to use standard solution algorithms, such as Sims (2001).

Consider the three equations NK model in $(53) \sim(55)$ and reported below as equations $(101) \sim(105)$

$$
\begin{aligned}
x_{t} & =E_{t}\left(x_{t+1}\right)-\tau\left(R_{t}-E_{t}\left(x_{t+1}\right)\right) \\
\pi_{t} & =\beta E_{t-1}\left(\pi_{t+1}\right)+\kappa x_{t} \\
R_{t} & =\psi \pi_{t}+\varepsilon_{R, t} \\
\eta_{1, t} & =x_{t}-E_{t-1}\left(x_{t}\right) \\
\eta_{2, t} & =\pi_{t}-E_{t-1}\left(\pi_{t}\right)
\end{aligned}
$$

The LRE model can be written in the following matrix form

$$
\Gamma_{0} X_{t}=\Gamma_{1} X_{t-1}+\Psi z_{t}+\Pi \eta_{t}
$$

where $X_{t}=\left(x_{t}, \pi_{t}, E_{t}\left(x_{t+1}\right), E_{t}\left(\pi_{t+1}\right)\right)^{T}, \varepsilon_{t}=\left(\varepsilon_{R, t}\right)$ and $\eta_{t}=\left(\eta_{1, t}, \eta_{2, t}\right)^{T}$.

The solution to (106) can be found following four steps. First, since the matrix $\Gamma_{0}$ is non-singular, the LRE model in (106) can be written as

$$
X_{t}=\Gamma_{1}^{*} X_{t-1}+\Psi^{*} \varepsilon_{t}+\Pi^{*} \eta_{t}
$$

where

$$
\begin{gathered}
\Gamma_{1}^{*} \equiv \Gamma_{0}^{-1} \Gamma_{1}=\left[\begin{array}{cc}
\mathbf{0}_{\mathbf{2} \times \mathbf{2}} & \mathbf{I}_{\mathbf{2} \times \mathbf{2}} \\
\mathbf{0}_{\mathbf{2} \times \mathbf{2}} & A_{2 \times 2}
\end{array}\right], \quad A \equiv\left[\begin{array}{cc}
1+\frac{\kappa \tau}{\beta} & \tau\left(\psi-\frac{1}{\beta}\right) \\
-\frac{\kappa}{\beta} & \frac{1}{\beta}
\end{array}\right] \\
\Psi^{*} \equiv \Gamma_{0}^{-1} \Psi=\left[\begin{array}{l}
0 \\
0 \\
\tau \\
0
\end{array}\right], \quad \Pi^{*} \equiv \Gamma_{0}^{-1} \Pi=\left[\begin{array}{c}
\mathbf{I}_{\mathbf{2} \times \mathbf{2}} \\
A_{2 \times 2}
\end{array}\right]
\end{gathered}
$$


Equivalently, the equations in (43) are

$$
\begin{aligned}
x_{t} & =E_{t-1}\left(x_{t}\right)+\eta_{1, t} \\
\pi_{t} & =E_{t-1}\left(\pi_{t}\right)+\eta_{2, t} \\
\xi_{t} & =A \xi_{t-1}+\left[\begin{array}{l}
\tau \\
0
\end{array}\right] \varepsilon_{R, t}+A \eta_{t}
\end{aligned}
$$

where $\xi_{t}=\left(E_{t}\left(x_{t+1}\right), E_{t}\left(\pi_{t+1}\right)\right)^{T}$.

Second, in order to study the stability of the system, the matrix $A$ is decomposed using the Jordan decomposition ${ }^{21}$ and (110) can be written as

$$
J^{-1} \xi_{t}=\Lambda J^{-1} \xi_{t-1}+J^{-1}\left[\begin{array}{l}
\tau \\
0
\end{array}\right] \varepsilon_{R, t}+J^{-1} A \eta_{t},
$$

where

$$
J^{-1}=\left[\begin{array}{cc}
-\frac{\kappa}{\phi} & -\frac{a_{2}}{2 \phi} \\
\frac{\kappa}{\phi} & \frac{\beta+\phi+\kappa \tau-1}{2 \phi}
\end{array}\right]
$$

and

$$
\Lambda=\left[\begin{array}{cc}
\lambda_{1} & 0 \\
0 & \lambda_{2}
\end{array}\right], \quad \lambda_{1,2}=\frac{(1+\beta+\kappa \tau) \pm \phi}{2 \beta}
$$

where $a_{2} \equiv(\beta-\phi+\kappa \tau-1), \phi \equiv\left[(1+\beta+\kappa \tau)^{2}-4 \beta(1+\kappa \tau \psi)\right]^{-1 / 2}$ and the diagonal elements of the matrix $\Lambda$ are the roots of the system and under determinacy $\left|\lambda_{1,2}\right|>1$.

Third, restrictions which eliminate the explosive dynamics of the system have to be imposed. Under determinacy both roots of (111) are unstable, which requires to impose the following conditions

$$
\begin{gathered}
\xi_{t}=\left(\begin{array}{l}
E_{t}\left(x_{t+1}\right) \\
E_{t}\left(\pi_{t+1}\right)
\end{array}\right)=\mathbf{0}_{\mathbf{2} \times \mathbf{1}} \\
\eta_{t}=-A^{-1}\left[\begin{array}{l}
\tau \\
0
\end{array}\right] \varepsilon_{R, t}=-\frac{\tau}{1+\kappa \tau \psi}\left[\begin{array}{l}
1 \\
\kappa
\end{array}\right] \varepsilon_{R, t}
\end{gathered}
$$

Fourth, the restrictions imposed on the endogenous variables and on the forecast errors are combined with the equations which define the remaining endogenous variables, that is (108) and (109). This implies

\footnotetext{
${ }^{21}$ The Jordan decomposition of the matrix $A$ is $A \equiv J \Lambda J^{-1}$, where the diagonal elements of the matrix $\Lambda$ are the roots of the system.
} 


$$
\left(\begin{array}{c}
x_{t} \\
\pi_{t}
\end{array}\right)=\eta_{t}=-\frac{\tau}{1+\kappa \tau \psi}\left[\begin{array}{l}
1 \\
\kappa
\end{array}\right] \varepsilon_{R, t} .
$$

b) Here we provide the derivation for the solution in Section 4.1 for the methodology proposed in this paper.

The proposed methodology consists in appending to the original LRE model the following equa$\operatorname{tion}^{22}$

$$
\omega_{t}=\frac{1}{\alpha} \omega_{t-1}+\nu_{t}-\eta_{2, t}
$$

where without loss of generality $\alpha \equiv \psi>1$. Denoting the newly defined vector of endogenous variables $\hat{X}_{t} \equiv\left(X_{t}, \omega_{t}\right)^{T}=\left(x_{t}, \pi_{t}, E_{t}\left(x_{t+1}\right), E_{t}\left(\pi_{t+1}\right), \omega_{t}\right)^{T}$, and the newly defined vector of exogenous shocks $\hat{\varepsilon}_{t} \equiv\left(\varepsilon_{t}, v_{t}\right)^{T}=\left(\varepsilon_{R, t}, v_{t}\right)^{T}$, the augmented representation of the LRE model is

$$
\hat{\Gamma}_{0} \hat{X}_{t}=\hat{\Gamma}_{1} \hat{X}_{t-1}+\hat{\Psi} \hat{z}_{t}+\hat{\Pi} \eta_{t}
$$

Given (116), the same steps are followed to obtain the solution to the system. First, the system in (116) is pre-multiplied by $\hat{\Gamma}_{0}^{-1}$ to obtain

$$
\hat{X}_{t}=\hat{\Gamma}_{1}^{*} \hat{X}_{t-1}+\hat{\Psi}^{*} \hat{\varepsilon}_{t}+\hat{\Pi}^{*} \eta_{t}
$$

where

$$
\hat{\Gamma}_{1}^{*} \equiv\left[\begin{array}{cc}
\Gamma_{1}^{*} & \mathbf{0}_{\mathbf{4} \times \mathbf{1}} \\
\mathbf{0}_{\mathbf{1} \times \mathbf{4}} & \frac{1}{\alpha}
\end{array}\right], \quad \hat{\Psi}^{*} \equiv\left[\begin{array}{cc}
\Psi^{*} & \mathbf{0}_{\mathbf{4} \times \mathbf{1}} \\
0 & -1
\end{array}\right], \quad \hat{\Pi}^{*} \equiv\left[\begin{array}{c}
\Pi_{4 \times 2}^{*} \\
0 \\
0
\end{array}\right] .
$$

\footnotetext{
${ }^{22}$ Note that $m=1$, thus implying that only one equation should be appended. Also, since Farmer et al. (2015) show that the choice of which forecast errors should be redefined as fundamental, it is without loss of generality that we consider the case when $\eta_{2, t}$ is redefined.
} 
Hence, defining $\hat{\xi}_{t} \equiv\left(\xi_{t}, \omega_{t}\right)^{T}=\left(E_{t}\left(x_{t+1}\right), E_{t}\left(\pi_{t+1}\right), \omega_{t}\right)^{T}$, the equations in (117) can be written as

$$
\begin{aligned}
& x_{t}=E_{t-1}\left(x_{t}\right)+\eta_{1, t} \\
& \pi_{t}=E_{t-1}\left(\pi_{t}\right)+\eta_{2, t}
\end{aligned}
$$

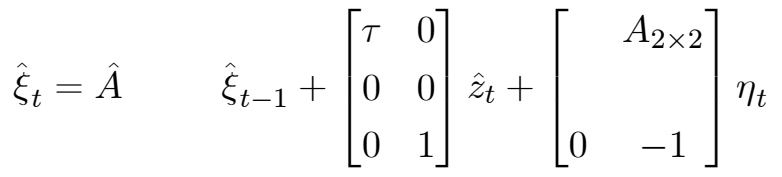

where $\hat{A}=\left[\begin{array}{cc}A & 0 \\ 0 & \alpha\end{array}\right]$.

Second, the matrix $\hat{A}$ is decomposed using the Jordan decomposition and the system in (120) can be written as

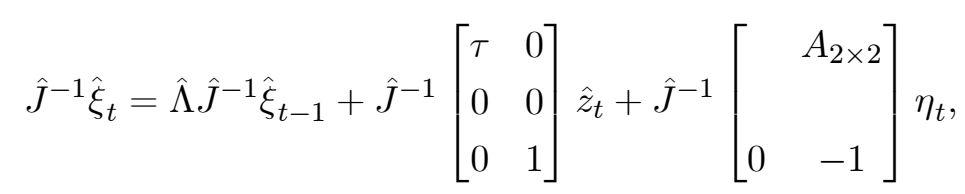

where

$$
\hat{J}^{-1} \equiv\left[\begin{array}{cc}
\mathbf{0}_{\mathbf{1} \times \mathbf{2}} & 1 \\
& \\
J^{-1} & \mathbf{0}_{\mathbf{2} \times \mathbf{1}}
\end{array}\right], \quad \hat{\Lambda} \equiv\left[\begin{array}{cc}
\lambda_{3} & 0 \\
0 & \Lambda
\end{array}\right]=\left[\begin{array}{ccc}
\lambda_{3} & & 0 \\
& \lambda_{1} & \\
0 & & \lambda_{2}
\end{array}\right]
$$

and $\lambda_{1,2}$ are the same as in $(112)$ and $\lambda_{3}=(1 / \alpha)=(1 / \psi)<1$.

Third, since $\left|\lambda_{1,2}\right|>1$ and $\lambda_{3}<1$, then the conditions which guarantee the boundedness of the solution are imposed on the last two equations of (121). This implies

$$
\begin{gathered}
\xi_{t}=\left(\begin{array}{c}
E_{t}\left(x_{t+1}\right) \\
E_{t}\left(\pi_{t+1}\right)
\end{array}\right)=\mathbf{0}_{\mathbf{2} \times \mathbf{1}} \\
\eta_{t}=-\frac{\tau}{1+\kappa \tau \psi}\left[\begin{array}{cc}
1 & 0 \\
\kappa & 0
\end{array}\right]\left[\begin{array}{c}
\varepsilon_{R, t} \\
\nu_{t}
\end{array}\right]
\end{gathered}
$$

Fourth, combining these restrictions with the first equation of (121) which displays stable dynamics and with (118) and (119), the obtained solution is

$$
\omega_{t}=\frac{1}{\alpha} \omega_{t-1}+\left[\frac{\tau \kappa}{1+\kappa \tau \psi} \quad 1\right]\left[\begin{array}{c}
\varepsilon_{R, t} \\
\nu_{t}
\end{array}\right]
$$




$$
\left(\begin{array}{c}
x_{t} \\
\pi_{t}
\end{array}\right)=\eta_{t}=-\frac{\tau}{1+\kappa \tau \psi}\left[\begin{array}{cc}
1 & 0 \\
\kappa & 0
\end{array}\right]\left[\begin{array}{c}
\varepsilon_{R, t} \\
\nu_{t}
\end{array}\right]
$$

\subsection{Appendix D}

In Section 4.2, the NK model is indeterminate and the derivations for the solutions under two alternative representations are provided.

c) To select a unique, bounded rational expectation equilibrium, we follow the solution method suggested by Farmer et al. (2015) when the forecast error for the deviations of inflation from its steady state is included as newly defined fundamental shock. Defining $\tilde{\varepsilon}_{t}=\left(\varepsilon_{t}, \eta_{2, t}\right)^{T}$, then the LRE can be written as

$$
\Gamma_{0} X_{t}=\Gamma_{1} X_{t-1}+\Psi_{f} \tilde{\varepsilon}_{t}+\Pi_{n} \eta_{1, t}
$$

The same steps as in Section 8.2 are also applied here. First, by pre-multiplying (126) by $\Gamma_{0}^{-1}$, we obtain the following equations

$$
\begin{aligned}
& x_{t}=E_{t-1}\left(x_{t}\right)+\eta_{1, t} \\
& \pi_{t}=E_{t-1}\left(\pi_{t}\right)+\eta_{2, t} \\
& \xi_{t}=A \xi_{t-1}+\left[\begin{array}{cc}
\tau & \tau\left(\psi-\frac{1}{\beta}\right) \\
0 & \frac{1}{\beta}
\end{array}\right] \tilde{\varepsilon}_{t}+\left[\begin{array}{c}
1+\frac{\kappa \tau}{\beta} \\
-\frac{\kappa}{\beta}
\end{array}\right] \eta_{1 t}
\end{aligned}
$$

where the matrix $A$ is the same as for the determinate case as defined in (110) and therefore also its Jordan decomposition delivers the same matrices $J$ and $\Lambda$ as in (111) and (112) and reported below

$$
\Lambda=\left[\begin{array}{cc}
\lambda_{1} & 0 \\
0 & \lambda_{2}
\end{array}\right], \quad \lambda_{1,2}=\frac{(1+\beta+\kappa \tau) \pm \phi}{2 \beta}
$$

and

$$
J^{-1}=\left[\begin{array}{cc}
-\frac{\kappa}{\phi} & -\frac{a_{2}}{2 \phi} \\
\frac{\kappa}{\phi} & \frac{\beta+\phi+\kappa \tau-1}{2 \phi}
\end{array}\right] .
$$

However, the difference with the determinate case is that, while in the latter both roots are outside the unit circle, under indeterminacy it is the case that $\left|\lambda_{1}\right|>1$ and $\left|\lambda_{2}\right|<1$. This implies that in the third step the restrictions imposed on the system to guarantee a bounded 
solution are also distinct from the determinate case. In particular, the restrictions are imposed on the first equation of (129), thus obtaining the following conditions

$$
\begin{gathered}
E_{t}\left(x_{t+1}\right)=-\frac{a_{2}}{2 \kappa} E_{t}\left(\pi_{t+1}\right) \\
\eta_{1, t}=\left[\begin{array}{ll}
-\frac{2 \beta \tau}{a_{3}} & \frac{2 \kappa \tau(1-\beta \psi)-a_{2}}{a_{3} \kappa}
\end{array}\right]\left[\begin{array}{l}
\varepsilon_{R, t} \\
\eta_{2, t}
\end{array}\right]
\end{gathered}
$$

where $a_{1}=(\beta-\phi+\kappa \tau+1), a_{2}=\left(a_{1}-2\right), a_{3}=\left(a_{1}+2 \phi\right)$ and $\phi=\left[(1+\beta+\kappa \tau)^{2}-4 \beta(1+\kappa \tau \psi)\right]^{-1 / 2}$. Fourth, using these restrictions, the solution obtained with the methodology of Farmer et al. (2015) is

$$
\left(\begin{array}{c}
x_{t} \\
\pi_{t} \\
E_{t}\left(x_{t+1}\right) \\
E_{t}\left(\pi_{t+1}\right)
\end{array}\right)=\underset{4 \times 1}{G} E_{t-1}\left(\pi_{t}\right)+\underset{4 \times 2}{H}\left[\begin{array}{c}
\varepsilon_{R, t} \\
\eta_{2, t}
\end{array}\right]
$$

where

$$
\underset{4 \times 1}{G} \equiv\left(\begin{array}{c}
-\frac{a_{2}}{2 \kappa} \\
1 \\
-\frac{a_{1} a_{2}}{4 \beta \kappa} \\
\frac{a_{1}}{2 \beta}
\end{array}\right) \quad \underset{4 \times 2}{H} \equiv\left(\begin{array}{cc}
-\frac{2 \beta \tau}{a_{3}} & \frac{2 \kappa \tau(1-\beta \psi)-a_{2}}{a_{3} \kappa} \\
0 & 1 \\
-\frac{\tau a_{2}}{a_{3}} & -\frac{a_{2}(1+\kappa \tau \psi)}{a_{3} \kappa} \\
\frac{2 \kappa \tau}{a_{3}} & -\frac{2(1+\kappa \tau \psi)}{a_{3}}
\end{array}\right)
$$

d) The derivation of the solution provided by the proposed methodology when the model is indeterminate closely follows the one described in Appendix B, part b). In particular, the first two steps of the solution method are equivalent and, recalling the definition of $\hat{\xi}_{t} \equiv\left(\xi_{t}, \omega_{t}\right)^{T}=\left(E_{t}\left(x_{t+1}\right), E_{t}\left(\pi_{t+1}\right), \omega_{t}\right)^{T}$ and $\hat{\varepsilon}_{t} \equiv\left(\varepsilon_{t}, v_{t}\right)^{T}=\left(\varepsilon_{R, t}, v_{t}\right)^{T}$, equation (121) is reported below as (134)

$$
\hat{J}^{-1} \hat{\xi}_{t}=\hat{\Lambda} \hat{J}^{-1} \hat{\xi}_{t-1}+\hat{J}^{-1}\left[\begin{array}{ll}
\tau & 0 \\
0 & 0 \\
0 & 1
\end{array}\right] \hat{\varepsilon}_{t}+\hat{J}^{-1}\left[\begin{array}{cc}
A_{2 \times 2} & 0 \\
& \\
0 & -1
\end{array}\right] \eta_{t}
$$

where

$$
\hat{J}^{-1} \equiv\left[\begin{array}{cc}
\mathbf{0}_{\mathbf{1} \times \mathbf{2}} & 1 \\
& \\
J^{-1} & \mathbf{0}_{\mathbf{2} \times \mathbf{1}}
\end{array}\right], \quad \hat{\Lambda}=\left[\begin{array}{cc}
\lambda_{3} & 0 \\
0 & \Lambda
\end{array}\right]=\left[\begin{array}{ccc}
\lambda_{3} & & 0 \\
& \lambda_{1} & \\
0 & & \lambda_{2}
\end{array}\right]
$$


It is however important to note that under indeterminacy not only $\left|\lambda_{1}\right|>1$ and $\left|\lambda_{2}\right|<1$ as in representation c), but also $\left|\lambda_{3}\right|=(1 / \alpha)=(1 / \psi)>1$. Hence, the third steps imposes restrictions on the first two equations of (134), which result in the following conditions

$$
\begin{gathered}
\omega_{t}=0 \\
E_{t}\left(x_{t+1}\right)=-\frac{a_{2}}{2 \kappa} E_{t}\left(\pi_{t+1}\right) \\
\eta_{t}=\left[\begin{array}{cc}
-\frac{2 \beta \tau}{a_{3}} & \frac{2 \kappa \tau(1-\beta \psi)-a_{2}}{a_{3} \kappa} \\
0 & 1
\end{array}\right]\left[\begin{array}{c}
\varepsilon_{R, t} \\
\nu_{t}
\end{array}\right]
\end{gathered}
$$

Fourth, using these restrictions, the solution of the LRE model for the endogenous variables takes the following form

$$
\left(\begin{array}{c}
x_{t} \\
\pi_{t} \\
E_{t}\left(x_{t+1}\right) \\
E_{t}\left(\pi_{t+1}\right)
\end{array}\right)=\underset{4 \times 1}{G} E_{t-1}\left(\pi_{t}\right)+\underset{4 \times 2}{H}\left[\begin{array}{c}
\varepsilon_{R, t} \\
\nu_{t}
\end{array}\right]
$$

